\title{
ON THE DESTRUCTION OF ION-SOUND WAVES IN PLASMA WITH STRONG SPACE-TIME DISPERSION
}

\author{
M. O. KORPUSOV
}

\begin{abstract}
The object of study in this paper is a model equation that describes ion-sound waves in plasma with the account of strong nonlinear dissipation and nonlinear sources of general type, together with strong space-time dispersion. Sufficient destruction conditions are obtained for the solution of the corresponding initial boundary problem in a bounded three-dimensional domain with homogeneous Dirichlet-Neumann conditions on the boundary. Moreover, the life-time of the solution is estimated. Finally, it is proved that, for every initial data in $\mathbb{H}_{0}^{2}(\Omega)$, a strong generalized solution of this problem exists locally in time, i.e., it is shown that the solution destruction time is always positive.
\end{abstract}

\section{$\S 1$. INTRODUCTION}

We continue the study of nonlinear Sobolev equations with second derivative in time and a nonlinear operator at the first order derivative in time. Consider the following model equation:

$$
\frac{\partial^{2}}{\partial t^{2}}\left(-\triangle^{2} u+\triangle u\right)+\frac{\partial}{\partial t} \operatorname{div}\left(\phi_{1}(x,|\nabla u|) \nabla u\right)+\triangle u-\operatorname{div}\left(\phi_{2}(x,|\nabla u|) \nabla u\right)=0 .
$$

H. A. Levine's well-known method (see [13, 14, 3]) is not applicable to this equation, at least in the form it was exposed in [14] and [3], because of the summand

$$
\frac{\partial}{\partial t} \operatorname{div}\left(\phi_{1}(x,|\nabla u|) \nabla u\right) \text {. }
$$

We observe that three methods are known that enable us to study how destruction emerges. The first of them is the nonlinear capacity method by Pokhozhaev and Mitidieri [8], the second is Levine's energy method (see [13, 14, 3, 10, ), and, finally the third is the method of automodel regimes, based on various comparison tests and developed by Samarskiı̆, Galaktionov, Kurdyumov, and Mikhaŭlov [9] (see also [2]).

\section{$\S 2$. Statement of the Problem}

When considering quasistationary processes in plasma, we encounter the following dependence of the inductive capacity tensor on the wave vector $k \in \mathbb{R}^{3}$ and the frequency $\omega \in \mathbb{R}^{1}$ (see, e.g., [6] and [7]):

$$
\varepsilon(k, \omega)=|k|^{2}+1-\frac{\omega_{0}^{2}}{\omega^{2}} .
$$

This form of the inductive capacity tensor involves both the strong space dispersion of the medium (this is reflected by the dependence on the wave vector $k$ ) and time dispersion (this is reflected by the dependence on the frequency $\omega \in \mathbb{R}^{1}$ ). It should be noted that

2010 Mathematics Subject Classification. Primary 35Q60.

Key words and phrases. Destruction, equations of Sobolev type, nonlinear analysis.

Supported by RFBR, grant no. 11-01-12018-ofi-m-2011, and by the President program for the support of young doctors of science, MD-99.2009.1. 
(2.1) is the equation for a Fourier transform, and the inductive capacity operator itself looks like this:

$$
\widehat{\varepsilon} \cdot=-\triangle \cdot+\mathrm{I} \cdot+\omega_{0}^{2} \int_{0}^{t} d s(t-s) \cdot
$$

where the electric displacement vector $\mathbb{D}$ and the electric field strength $\mathbb{E}$ are related by the formula

$$
\mathbb{D}=\widehat{\varepsilon} \mathbb{E} .
$$

However, the inductive capacity operator $\widehat{\varepsilon}$ defined by (2.2) does not take the nonlinear properties of the medium into account. Therefore, our task is to generalize (2.2) to the nonlinear case. In fact, the general form of the equation relating the electric displacement vector in a medium and the electric field strength is

$$
\mathbb{D}=\mathbb{E}+4 \pi \mathbb{P},
$$

where $\mathbb{P}$ is the polarization vector of the medium. With the time and space dispersion taken into account, $\mathbb{P}$ depends on $\mathbb{E}$ in the following way:

$$
\mathbb{P}=-\triangle \mathbb{E}+\omega_{0}^{2} \int_{0}^{t} d \tau(t-\tau)[\mathbb{E}(\tau)-\varkappa(x,|\mathbb{E}|(\tau)) \mathbb{E}(\tau)]
$$

Here the function $\varkappa(x,|\mathbb{E}|)$ reflects (among other things) the Kerr dependence of the polarization vector on the field:

$$
\varkappa(x,|\mathbb{E}|)=\varkappa_{0}|\mathbb{E}|^{2}, \quad \varkappa_{0}>0 .
$$

Next, this function appears in (2.5) with the "minus" sign, which corresponds to the so-called defocusing medium (see, e.g., 6]). In the quasistationary approximation, the Maxwell system of equations for the electric field takes the form

$$
\operatorname{div} \mathbb{D}=-4 \pi n, \quad \operatorname{curl} \mathbb{E}=0,
$$

where $n$ is the concentration of free charges, which satisfies the equation

$$
\frac{\partial n}{\partial t}=\operatorname{div} \mathbb{J}, \quad \mathbb{J}=\sigma(x,|\mathbb{E}|) \mathbb{E} ;
$$

physically, here $\sigma(x,|\mathbb{E}|)$ is the conductivity of the medium (generally speaking, it may depend both on the point $x \in \Omega$ and on the modulus $|\mathbb{E}|$ of the electric field strength). Now, we make some assumptions about the properties of the domain $\Omega \subset \mathbb{R}^{3}$ in which the system (2.4) - (2.7) of medium and field equations is considered. We assume that $\Omega \subset \mathbb{R}^{3}$ is a bounded simply connected domain with a $\mathbb{C}^{4, \delta}$-boundary $\partial \Omega$ for some $\delta \in(0,1]$. Since $\Omega$ is simply connected, the equation curl $\mathbb{E}=0$ implies the existence of an electric potential:

$$
\mathbb{E}(x)=-\nabla u(x), \quad x=\left(x_{1}, x_{2}, x_{3}\right) \in \Omega .
$$

But then equations (2.4)-(2.6) imply the formula

$$
-4 \pi \triangle^{2} u+\triangle u+4 \pi \omega_{0}^{2} \int_{0}^{t}(t-\tau)[\triangle u(\tau)+\operatorname{div}(\varkappa(x,|\nabla u|) \nabla u)(\tau)]=4 \pi n,
$$

and equation (2.7) yields

$$
\frac{\partial n}{\partial t}=-\operatorname{div}(\sigma(x,|\nabla u|) \nabla u) .
$$

The last two formulas imply yet another equation:

$$
\begin{aligned}
\frac{\partial^{2}}{\partial t^{2}}\left(-4 \pi \triangle^{2} u+\triangle u\right) & +4 \pi \frac{\partial}{\partial t} \operatorname{div}(\sigma(x,|\nabla u|) \nabla u) \\
& +4 \pi \omega_{0}^{2} \triangle u-4 \pi \omega_{0}^{2} \operatorname{div}(\varkappa(x,|\nabla u|) \nabla u)=0 .
\end{aligned}
$$


In dimensionless variables, it takes the form

$$
\frac{\partial^{2}}{\partial t^{2}}\left(-\triangle^{2} u+\triangle u\right)+\frac{\partial}{\partial t} \operatorname{div}\left(\phi_{1}(x,|\nabla u|) \nabla u\right)+\triangle u-\operatorname{div}\left(\phi_{2}(x,|\nabla u|) \nabla u\right)=0,
$$

where

$$
\begin{aligned}
\triangle^{2} & \equiv \triangle \triangle, \quad \triangle=\left(\frac{\partial^{2}}{\partial x_{1}^{2}}, \frac{\partial^{2}}{\partial x_{2}^{2}}, \frac{\partial^{2}}{\partial x_{3}^{2}}\right), \\
x & =\left(x_{1}, x_{2}, x_{3}\right), \quad|\nabla u|=\sqrt{u_{x_{1}}^{2}+u_{x_{2}}^{2}+u_{x_{3}}^{2}}, \quad x \in \Omega \subset \mathbb{R}^{3} .
\end{aligned}
$$

Physically, $u=u(x, t)$ is an electric potential. Thus, if we assume that $\partial \Omega$ is a "grounded ideal conductor", we arrive at the Dirichlet-Neumann boundary conditions

$$
\left.u\right|_{\partial \Omega}=\left.\frac{\partial u}{\partial n_{x}}\right|_{\partial \Omega}=0 .
$$

Furthermore, we supplement equation (2.11) with the initial conditions

$$
u(x, 0)=u_{0}(x), \quad u^{\prime}(x, 0)=u_{1}(x), \quad u_{0}(x), u_{1}(x) \in \mathbb{H}_{0}^{2}(\Omega) ;
$$

here and below, $v^{\prime}$ denotes the partial derivative with respect to time.

\section{§3. Destruction of a strong Generalized solution}

First, we introduce some conditions on the functions

$$
\phi_{1}(x, s), \phi_{2}(x, s): \Omega \otimes \mathbb{R}_{+}^{1} \rightarrow \mathbb{R}^{1} .
$$

We recall the definition of a Carathéodory function.

Definition 1. A mapping $f(x, s): \Omega \otimes \mathbb{R}_{+}^{1} \rightarrow \mathbb{R}^{1}$ is called a Carathéodory function if it is continuous in $s$ for a.e. $x \in \Omega$ and measurable in $x \in \Omega$ for all $s \in \mathbb{R}_{+}^{1}$.

We impose the following conditions on the function $\phi_{1}(x, s): \Omega \otimes \mathbb{R}_{+}^{1} \rightarrow \mathbb{R}_{+}^{1}$.

Conditions on $\phi_{1}(x, s)$.

(i) $\phi_{1}(x, s)$ is a Carathéodory function;

(ii) ${ }_{1}$ for a.e. $x \in \Omega$, the function $\phi_{1}(x, s)$ satisfies the following growth conditions:

$$
\left|\phi_{1}(x, s)\right| \leq c_{1}+c_{2}|s|^{p_{1}-2} \quad \text { for } \quad p_{1} \in(2,4] ;
$$

for a.e. $x \in \Omega, \phi_{1}(x, s)$ belongs to $\mathbb{C}^{(1)}([0,+\infty))$ as a function of $s$, and

$$
\begin{array}{ll}
\left|s \phi_{1 s}^{\prime}(x, s)\right| \leq c_{1}+c_{2}|s|^{p_{1}-2} & \text { for } p_{1} \in(2,4], \\
\phi(x, s) \geq 0, \quad \phi_{1 s}^{\prime}(x, s) \geq 0 & \text { for all } s \in \mathbb{R}_{+}^{1} \quad \text { and a.e. } \quad x \in \Omega ;
\end{array}
$$

(iii) $)_{1}$ for every $v \in \mathbb{W}_{0}^{1, p_{1}}(\Omega)$, the operator $\operatorname{div}\left(\phi_{1}(x,|\nabla v|) \nabla v\right)$ is locally Lipschitz continuous and Fréchet differentiable:

$$
\left\|\operatorname{div}\left(\phi_{1}\left(x,\left|\nabla v_{1}\right|\right) \nabla v_{1}\right)-\operatorname{div}\left(\phi_{1}\left(x,\left|\nabla v_{2}\right|\right) \nabla v_{2}\right)\right\|_{-1, p_{1}^{\prime}} \leq \mu_{1}\left(\mathrm{R}_{1}\right)\left\|\nabla v_{1}-\nabla v_{2}\right\|_{p_{1}}
$$

for all $v_{1}, v_{2} \in \mathbb{W}_{0}^{1, p_{1}}(\Omega)$, where $p_{1}^{\prime}=p_{1} /\left(p_{1}-1\right),\|\cdot\|_{-1, p_{1}^{\prime}}$ is the norm of the Banach space $\mathbb{W}^{-1, p_{1}^{\prime}}(\Omega),\|\cdot\|_{p_{1}}$ is the norm of the Banach space $\mathbb{L}^{p_{1}}(\Omega)$,

$$
\mathrm{R}_{1}=\max \left\{\left\|\nabla v_{1}\right\|_{p_{1}},\left\|\nabla v_{2}\right\|_{p_{1}}\right\}
$$

and $\mu_{1}(\cdot)$ is a nonnegative and monotone nondecreasing function. 
Remark 1. The fact that the same constants $c_{1}, c_{2}>0$ occur both in (3.1) and (3.2) is not a strong restriction. We have taken them to be the same only to shorten unnecessarily bulky formulas. Note that, for instance, the function $\phi_{1}(x, s)=s^{p_{1}-2}$ satisfies the above conditions if $p_{1} \in[3,4]$. For this function, the operator

$$
\operatorname{div}\left(\phi_{1}(x,|\nabla u|) \nabla u\right)=\operatorname{div}\left(|\nabla u|^{p_{1}-2} \nabla u\right) \quad \text { for } \quad p_{1} \in[3,4]
$$

is the classical pseudo-Laplacian.

Now, we introduce some assumptions about $\phi_{2}(x, s): \Omega \otimes \mathbb{R}_{+}^{1} \rightarrow \mathbb{R}^{1}$.

Conditions on $\phi_{2}(x, s)$.

(i) $\phi_{2}(x, s)$ is a Carathéodory function;

(ii) ${ }_{2}$ it satisfies the growth condition

$$
\left|\phi_{2}(x, s)\right| \leq c_{3}+c_{4}|s|^{p_{2}-2} \quad \text { for a.e. } \quad x \in \Omega \quad \text { and } \quad p_{2} \in(2,6] ;
$$

(iii) $)_{2}$ there is $\vartheta>2$ such that for all $v(x) \in \mathbb{W}_{0}^{1, p_{2}}(\Omega)$ we have

$$
\int_{\Omega}|\nabla v|^{2} \phi_{2}(x,|\nabla v|) d x \geq \vartheta \int_{\Omega} d x \int_{0}^{|\nabla v|} s \phi_{2}(x, s) d s ;
$$

(iv) $)_{2}$ for all $v \in \mathbb{W}_{0}^{1, p_{1}}(\Omega)$, the operator $\operatorname{div}\left(\phi_{1}(x,|\nabla v|) \nabla v\right)$ is locally Lipschitz-continuous:

$$
\left\|\operatorname{div}\left(\phi_{2}\left(x,\left|\nabla v_{1}\right|\right) \nabla v_{1}\right)-\operatorname{div}\left(\phi_{2}\left(x,\left|\nabla v_{2}\right|\right) \nabla v_{2}\right)\right\|_{-1, p_{2}^{\prime}} \leq \mu_{2}\left(\mathrm{R}_{2}\right)\left\|\nabla v_{1}-\nabla v_{2}\right\|_{p_{2}}
$$

for all $v_{1}, v_{2} \in \mathbb{W}_{0}^{1, p_{2}}(\Omega)$, where $p_{2}^{\prime}=p_{2} /\left(p_{2}-1\right),\|\cdot\|_{-1, p_{2}^{\prime}}$ is the norm in the Banach space $\mathbb{W}^{-1, p_{2}^{\prime}}(\Omega),\|\cdot\|_{p_{2}}$ is the norm in the Banach space $\mathbb{L}^{p_{2}}(\Omega)$,

$$
\mathrm{R}_{2}=\max \left\{\left\|\nabla v_{1}\right\|_{p_{2}},\left\|\nabla v_{2}\right\|_{p_{2}}\right\},
$$

and $\mu_{2}(\cdot)$ is a nonnegative monotone nondecreasing function.

The constants $c_{1}, c_{2}, c_{3}, c_{4}$ are positive.

Before giving the definition of a strong generalized solution of the problem, we need to discuss the properties of the operators

$$
\operatorname{div}\left(\phi_{1}(x,|\nabla v|) \nabla v\right) \text { and } \operatorname{div}\left(\phi_{2}(x,|\nabla v|) \nabla v\right) .
$$

For this, we recall the notion of a Nemytskil operator.

Definition 2. For a Carathéodory function $f(x, u)$, the operator $N_{f}(u) \equiv f(x, u)$ is called a Nemytskiı̌ operator.

We prove that, under the conditions $(\mathrm{i})_{1}-(\mathrm{ii})_{1}$ and $(\mathrm{i})_{2}-(\mathrm{ii})_{2}$ these operators act, respectively, as follows:

$$
\begin{aligned}
& \operatorname{div}\left(\phi_{1}(x,|\nabla v|) \nabla v\right): \mathbb{W}_{0}^{1, p_{1}}(\Omega) \rightarrow \mathbb{W}^{-1, p_{1}^{\prime}}(\Omega), \\
& \operatorname{div}\left(\phi_{2}(x,|\nabla v|) \nabla v\right): \mathbb{W}_{0}^{1, p_{2}}(\Omega) \rightarrow \mathbb{W}^{-1, p_{2}^{\prime}}(\Omega),
\end{aligned}
$$

where $p_{1}^{\prime}=p_{1} /\left(p_{1}-1\right)$ and $p_{2}^{\prime}=p_{2} /\left(p_{2}-1\right)$. Indeed, consider the first of them (the second is treated similarly). Let $v \in \mathbb{W}_{0}^{1, p_{1}}(\Omega)$ with $p_{1} \in(2,4]$. Then

$$
\eta=\nabla v: \mathbb{W}_{0}^{1, p_{1}}(\Omega) \rightarrow \mathbb{L}^{p_{1}}(\Omega) \otimes \mathbb{L}^{p_{1}}(\Omega) \otimes \mathbb{L}^{p_{1}}(\Omega) .
$$

Consider the vector-valued function

$$
f(x, \eta) \equiv \phi_{1}(x,|\eta|) \eta .
$$

By $(i)_{1}, \phi_{1}(x, s): \Omega \otimes \mathbb{R}_{+}^{1} \rightarrow \mathbb{R}^{1}$ is a Carathéodory function. Then $\phi_{1}(x,|\eta|)$, when viewed as a mapping

$$
\phi_{1}(x,|\eta|): \Omega \otimes \mathbb{R}^{3} \rightarrow \mathbb{R}^{1},
$$


is also a Carathéodory function. Thus, the product $\phi_{1}(x,|\eta|) \eta$ is also a Carathéodory function of $\eta=\left(\eta_{1}, \eta_{2}, \eta_{3}\right)$. So, we have proved that

$$
f(x, \eta) \equiv \phi_{1}(x,|\eta|) \eta: \Omega \otimes \mathbb{R}^{3} \rightarrow \mathbb{R}^{3}
$$

is a Carathéodory function. Now, we obtain a growth estimate for $f(x, \eta)$ :

$$
\begin{aligned}
|f(x, \eta)| & \leq\left|\phi_{1}(x,|\eta|)\right||\eta| \leq c_{1}|\eta|+c_{2}|\eta|^{p_{1}-1} \\
& \leq \frac{|\eta|^{p_{1}-1}}{p_{1}-1}+\frac{p_{1}-2}{p_{1}-1} c_{1}^{\left(p_{1}-1\right) /\left(p_{1}-2\right)}+c_{2}|\eta|^{p_{1}-1}=\bar{c}_{1}+\bar{c}_{2}|\eta|^{p_{1}-1},
\end{aligned}
$$

where

$$
\bar{c}_{1}=\frac{p_{1}-2}{p_{1}-1} c_{1}^{\left(p_{1}-1\right) /\left(p_{1}-2\right)}, \quad \bar{c}_{2}=c_{2}+\frac{1}{p_{1}-1} .
$$

So, $f(x, \eta)$ is a Carathéodory function and satisfies the growth condition (3.10). This means that the corresponding Nemytskil operator acts in the following way:

(3.11) $\xi=N_{f}(\eta): \mathbb{L}^{p_{1}}(\Omega) \otimes \mathbb{L}^{p_{1}}(\Omega) \otimes \mathbb{L}^{p_{1}}(\Omega) \rightarrow \mathbb{L}^{p_{1}^{\prime}}(\Omega) \otimes \mathbb{L}^{p_{1}^{\prime}}(\Omega) \otimes \mathbb{L}^{p_{1}^{\prime}}(\Omega), \quad p_{1}^{\prime}=\frac{p_{1}}{p_{1}-1}$,

and, by Krasnosel'skilı's theorem [4], it is a continuous mapping in the strong topologies of the corresponding Banach spaces

$$
\mathbb{B} \equiv \mathbb{L}^{p_{1}}(\Omega) \otimes \mathbb{L}^{p_{1}}(\Omega) \otimes \mathbb{L}^{p_{1}}(\Omega) \quad \text { and } \quad \mathbb{B}^{*} \equiv \mathbb{L}^{p_{1}^{\prime}}(\Omega) \otimes \mathbb{L}^{p_{1}^{\prime}}(\Omega) \otimes \mathbb{L}^{p_{1}^{\prime}}(\Omega) .
$$

It remains to observe that the operator $\operatorname{div} \cdot$, understood in the weak sense, acts as shown below:

$$
\operatorname{div} \xi: \mathbb{L}^{p_{1}^{\prime}}(\Omega) \otimes \mathbb{L}^{p_{1}^{\prime}}(\Omega) \otimes \mathbb{L}^{p_{1}^{\prime}}(\Omega) \rightarrow \mathbb{W}^{-1, p_{1}^{\prime}}(\Omega)
$$

Since

we see that

$$
\operatorname{div}\left(\phi_{1}(x,|\nabla v|) \nabla v\right)=\operatorname{div}\left(N_{f}(\nabla v)\right)
$$

$$
\operatorname{div}\left(\phi_{1}(x,|\nabla v|) \nabla v\right): \mathbb{W}_{0}^{1, p_{1}}(\Omega) \rightarrow \mathbb{W}^{-1, p_{1}^{\prime}}(\Omega) .
$$

Now, by (ii) $)_{1}$ and (ii) $)_{2}$, we have $p_{1} \in(2,4]$ and $p_{2} \in(2,6]$. Since the domain $\Omega \subset \mathbb{R}^{3}$ is bounded and the space $\mathbb{H}_{0}^{2}(\Omega)$ is reflexive, we arrive at the following two chains of dense and continuous embeddings:

$$
\begin{aligned}
& \mathbb{H}_{0}^{2}(\Omega) \stackrel{d s}{\subset} \mathbb{W}_{0}^{1, p_{1}}(\Omega) \stackrel{d s}{\subset} \mathbb{W}^{-1, p_{1}^{\prime}}(\Omega) \stackrel{d s}{\subset} \mathbb{H}^{-2}(\Omega), \\
& \mathbb{H}_{0}^{2}(\Omega) \stackrel{d s}{\subset} \mathbb{W}_{0}^{1, p_{2}}(\Omega) \stackrel{d s}{\subset} \mathbb{W}^{-1, p_{2}^{\prime}}(\Omega) \stackrel{d s}{\subset} \mathbb{H}^{-2}(\Omega) .
\end{aligned}
$$

We denote by $\langle\cdot, \cdot\rangle$ the pairing between $\mathbb{H}_{0}^{2}(\Omega)$ and $\mathbb{H}^{-2}(\Omega)$, by $\langle\cdot, \cdot\rangle_{1}$ the pairing between $\mathbb{W}_{0}^{1, p_{1}}(\Omega)$ and $\mathbb{W}^{-1, p_{1}^{\prime}}(\Omega)$ and, finally, by $\langle\cdot, \cdot\rangle_{2}$ the pairing between $\mathbb{W}_{0}^{1, p_{2}}(\Omega)$ and $\mathbb{W}^{-1, p_{2}^{\prime}}(\Omega)$. Next, we consider the following embedding operators $\mathbb{J}_{1}$ and $\mathbb{J}_{2}$ :

$$
\mathbb{J}_{1}: \mathbb{H}_{0}^{2}(\Omega) \rightarrow \mathbb{W}_{0}^{1, p_{1}}(\Omega), \quad \mathbb{J}_{2}: \mathbb{H}_{0}^{2}(\Omega) \rightarrow \mathbb{W}_{0}^{1, p_{2}}(\Omega) .
$$

By (3.13) and (3.14), the corresponding transpose operators

$$
\mathbb{J}_{1}^{t}: \mathbb{W}^{-1, p_{1}^{\prime}}(\Omega) \rightarrow \mathbb{H}^{-2}(\Omega), \quad \mathbb{J}_{2}^{t}: \mathbb{W}^{-1, p_{2}^{\prime}}(\Omega) \rightarrow \mathbb{H}^{-2}(\Omega)
$$

defined by

$$
\begin{aligned}
& \left\langle\mathbb{J}_{1}^{t} f_{1}, w\right\rangle=\left\langle f_{1}, \mathbb{J}_{1} w\right\rangle_{1} \text { for all } f_{1} \in \mathbb{W}^{-1, p_{1}^{\prime}}(\Omega) \text { and all } w \in \mathbb{H}_{0}^{2}(\Omega), \\
& \left\langle\mathbb{J}_{2}^{t} f_{2}, w\right\rangle=\left\langle f_{2}, \mathbb{J}_{2} w\right\rangle_{2} \text { for all } f_{2} \in \mathbb{W}^{-1, p_{2}^{\prime}}(\Omega) \text { and all } w \in \mathbb{H}_{0}^{2}(\Omega),
\end{aligned}
$$

are also embeddings. So, if we identify $\mathbb{H}_{0}^{2}(\Omega)$ with $\mathbb{J}_{1} \mathbb{H}_{0}^{2}(\Omega) \subset \mathbb{W}_{0}^{1, p_{1}^{\prime}}(\Omega)$ and $\mathbb{H}_{0}^{2}(\Omega)$ with $\mathbb{J}_{2} \mathbb{H}_{0}^{2}(\Omega) \subset \mathbb{W}_{0}^{1, p_{2}^{\prime}}(\Omega)$, we also may identify $\mathbb{W}^{-1, p_{1}^{\prime}}(\Omega)$ with $\mathbb{J}_{1}^{t} \mathbb{W}^{-1, p_{1}^{\prime}}(\Omega) \subset \mathbb{H}^{-2}(\Omega)$, and 
$\mathbb{W}^{-1, p_{2}^{\prime}}(\Omega)$ with $\mathbb{J}_{2}^{t} \mathbb{W}^{-1, p_{2}^{\prime}}(\Omega) \subset \mathbb{H}^{-2}(\Omega)$. After these identifications, (3.15) and (3.16) turn into

$$
\begin{aligned}
& \left\langle f_{1}, w\right\rangle=\left\langle f_{1}, w\right\rangle_{1} \text { for all } f_{1} \in \mathbb{W}^{-1, p_{1}^{\prime}}(\Omega) \text { and all } w \in \mathbb{H}_{0}^{2}(\Omega), \\
& \left\langle f_{2}, w\right\rangle=\left\langle f_{2}, w\right\rangle_{2} \text { for all } f_{2} \in \mathbb{W}^{-1, p_{2}^{\prime}}(\Omega) \text { and all } w \in \mathbb{H}_{0}^{2}(\Omega) .
\end{aligned}
$$

Now, we are in a position to define a strong generalized solution of problem (2.11)(2.13).

Definition 3. A function $u(x, t) \mathbb{C}^{(2)}\left([0, \mathrm{~T}] ; \mathbb{H}_{0}^{2}(\Omega)\right)$ of class $\mathbb{C}^{(2)}\left([0, \mathrm{~T}] ; \mathbb{H}_{0}^{2}(\Omega)\right)$ for some $\mathrm{T}>0$ is called a strong generalized solution of problem (2.11)-(2.13) if

$$
\langle\mathrm{D}(u), w\rangle=0 \quad \text { for all } \quad t \in[0, \mathrm{~T}] \quad \text { and all } \quad w \in \mathbb{H}_{0}^{2}(\Omega)
$$

where

$$
\mathrm{D}(u) \equiv \frac{\partial^{2}}{\partial t^{2}}\left(-\triangle^{2} u+\triangle u\right)+\frac{\partial}{\partial t} \operatorname{div}\left(\phi_{1}(x,|\nabla u|) \nabla u\right)+\triangle u-\operatorname{div}\left(\phi_{2}(x,|\nabla u|) \nabla u\right),
$$

and $\langle\cdot, \cdot\rangle$ denotes the pairing between the Banach spaces $\mathbb{H}_{0}^{2}(\Omega)$ and $\mathbb{H}^{-2}(\Omega)$.

Remark 2. It should be noted that the operator $\triangle^{2}$ is understood in the following sense:

$$
\left\langle\triangle^{2} u, w\right\rangle \equiv \int_{\Omega} \triangle u \triangle w d x \quad \text { for all } \quad u, w \in \mathbb{H}_{0}^{2}(\Omega)
$$

When we calculate the distribution $\triangle^{2} u$ in accordance with the above identity, we shall refer to "integration by parts".

We introduce the following notation:

$$
\begin{aligned}
\Phi(t) & \equiv \Phi[u](t) \equiv \frac{1}{2} \int_{\Omega}|\triangle u|^{2} d x+\frac{1}{2} \int_{\Omega}|\nabla u|^{2} d x, \\
\mathrm{~J}(t) & \equiv \mathrm{J}[u](t) \equiv \int_{\Omega}\left|\triangle u^{\prime}\right|^{2} d x+\int_{\Omega}\left|\nabla u^{\prime}\right|^{2} d x
\end{aligned}
$$

Lemma 1. If $u(x, t) \in \mathbb{C}^{(1)}\left([0, \mathrm{~T}] ; \mathbb{H}_{0}^{1}(\Omega)\right)$ for some $\mathrm{T}>0$, then

$$
\left(\Phi^{\prime}\right)^{2}(t) \leq 2 \Phi(t) \mathrm{J}(t) \quad \text { for all } \quad t \in[0, \mathrm{~T}] .
$$

Proof. Since $u(x, t) \in \mathbb{C}^{(1)}\left([0, T] ; \mathbb{H}_{0}^{1}(\Omega)\right)$, we have

$$
\Phi^{\prime}=\int_{\Omega}\left(\nabla u^{\prime}, \nabla u\right) d x+\int_{\Omega} \triangle u^{\prime} \triangle u d x
$$

Next,

$$
\begin{aligned}
\left|\int_{\Omega}\left(\nabla u^{\prime}, \nabla u\right) d x\right| & \leq\left\|\nabla u^{\prime}\right\|_{2}\|\nabla u\|_{2}, \\
\left|\int_{\Omega} \triangle u^{\prime} \triangle u d x\right| & \leq\left(\int_{\Omega}\left|\triangle u^{\prime}\right|^{2} d x\right)^{1 / 2}\left(\int_{\Omega}|\triangle u|^{2} d x\right)^{1 / 2} .
\end{aligned}
$$


Therefore,

$$
\begin{aligned}
\left(\Phi^{\prime}\right)^{2} \leq & \left(\left\|\nabla u^{\prime}\right\|_{2}\|\nabla u\|_{2}+\left(\int_{\Omega}\left|\triangle u^{\prime}\right|^{2} d x\right)^{1 / 2}\left(\int_{\Omega}|\triangle u|^{2} d x\right)^{1 / 2}\right)^{2} \\
\leq & \left\|\nabla u^{\prime}\right\|_{2}^{2}\|\nabla u\|_{2}^{2}+\int_{\Omega}\left|\Delta u^{\prime}\right|^{2} d x \int_{\Omega}|\triangle u|^{2} d x \\
& +2\left\|\nabla u^{\prime}\right\|_{2}\|\nabla u\|_{2}\left(\int_{\Omega}\left|\triangle u^{\prime}\right|^{2} d x\right)^{1 / 2}\left(\int_{\Omega}|\triangle u|^{2} d x\right)^{1 / 2} \\
\leq & \left\|\nabla u^{\prime}\right\|_{2}^{2}\|\nabla u\|_{2}^{2}+\int_{\Omega}\left|\triangle u^{\prime}\right|^{2} d x \int_{\Omega}|\triangle u|^{2} d x \\
& \quad+\left\|\nabla u^{\prime}\right\|_{2}^{2} \int_{\Omega}|\triangle u|^{2} d x+\|\nabla u\|_{2}^{2} \int_{\Omega}\left|\triangle u^{\prime}\right|^{2} d x \\
= & \left(\left\|\nabla u^{\prime}\right\|_{2}^{2}+\int_{\Omega}\left|\triangle u^{\prime}\right|^{2} d x\right)\left(\|\nabla u\|_{2}^{2}+\int_{\Omega}|\triangle u|^{2} d x\right)=2 \mathrm{~J}(t) \Phi(t)
\end{aligned}
$$

where we have used the inequality $2 a b \leq a^{2}+b^{2}$ with

$$
a=\left\|\nabla u^{\prime}\right\|_{2}\left(\int_{\Omega}|\triangle u|^{2} d x\right)^{1 / 2}, \quad b=\|\nabla u\|_{2}\left(\int_{\Omega}\left|\triangle u^{\prime}\right|^{2} d x\right)^{1 / 2} .
$$

We pass to the deduction of the first and the second energy inequalities. Suppose that for some $\mathrm{T}>0$ problem (2.11)-(2.13) admits a strong generalized solution $u(x, t)$ of class $\mathbb{C}^{(2)}\left([0, \mathrm{~T}] ; \mathbb{H}_{0}^{2}(\Omega)\right)$, in the sense of Definition 3 .

We take the solution $u$ itself for the role of $w$ in (3.19): $w=u$. After "integration by parts", we obtain

$$
\begin{aligned}
& \int_{\Omega} \Delta u^{\prime \prime} \Delta u d x+\int_{\Omega}\left(\nabla u^{\prime \prime}, \nabla u\right) d x \\
& \quad+\int_{\Omega}\left(\nabla u, \frac{\partial}{\partial t}\left[\phi_{1}(x,|\nabla u|) \nabla u\right]\right) d x+\int_{\Omega}|\nabla u|^{2} d x=\int_{\Omega} \phi_{2}(x,|\nabla u|)|\nabla u|^{2} d x .
\end{aligned}
$$

Observe that

$$
\begin{aligned}
\int_{\Omega}\left(\nabla u^{\prime \prime}, \nabla u\right) d x & =\frac{d}{d t} \int_{\Omega}\left(\nabla u^{\prime}, \nabla u\right) d x-\left\|\nabla u^{\prime}\right\|_{2}^{2} \\
& =\frac{1}{2} \frac{d^{2}}{d t^{2}}\|\nabla u\|_{2}^{2}-\left\|\nabla u^{\prime}\right\|_{2}^{2} \\
\int_{\Omega} \triangle u^{\prime \prime} \triangle u d x & =\frac{d}{d t} \int_{\Omega} \triangle u^{\prime} \triangle u d x-\int_{\Omega}\left|\triangle u^{\prime}\right|^{2} d x \\
& =\frac{1}{2} \frac{d^{2}}{d t^{2}} \int_{\Omega}|\triangle u|^{2} d x-\int_{\Omega}\left|\triangle u^{\prime}\right|^{2} d x
\end{aligned}
$$

Consider the following integral separately:

$$
\begin{aligned}
\mathrm{I}_{1} & \equiv \int_{\Omega}\left(\nabla u, \frac{\partial}{\partial t}\left[\phi_{1}(x,|\nabla u|) \nabla u\right]\right) d x \\
& =\int_{\Omega} \phi_{1}(x,|\nabla u|)\left(\nabla u^{\prime}, \nabla u\right) d x+\left.\int_{\Omega}|\nabla u|^{2} \phi_{1 s}^{\prime}(x, s)\right|_{s=|\nabla u|} \frac{\partial}{\partial t}|\nabla u| d x \\
& =\mathrm{I}_{2}+\mathrm{I}_{3} .
\end{aligned}
$$


We transform the integral $\mathrm{I}_{3}$ :

$$
\begin{aligned}
\mathrm{I}_{3} & =\left.\int_{\Omega}|\nabla u|^{2} \phi_{1 s}^{\prime}(x, s)\right|_{s=|\nabla u|} \frac{\partial}{\partial t}|\nabla u| d x \\
& =\left.\int_{\Omega}|\nabla u| \phi_{1 s}^{\prime}(x, s)\right|_{s=|\nabla u|} \frac{1}{2} \frac{\partial}{\partial t}|\nabla u|^{2} d x \\
& =\left.\int_{\Omega}|\nabla u| \phi_{1 s}^{\prime}(x, s)\right|_{s=|\nabla u|}\left(\nabla u^{\prime}, \nabla u\right) d x \\
& =\int_{\Omega}\left(\nabla u^{\prime},\left.|\nabla u| \phi_{1 s}^{\prime}(x, s)\right|_{s=|\nabla u|} \nabla u\right) d x .
\end{aligned}
$$

For $\mathrm{I}_{2}$, we have

$$
\mathrm{I}_{2}=\int_{\Omega}\left(\nabla u^{\prime}, \phi_{1}(x,|\nabla u|) \nabla u\right) d x .
$$

Now, using the notation (3.20), (3.21), from (3.23) and the definitions of $\mathrm{I}_{2}$ and $\mathrm{I}_{3}$ we deduce the formula

$$
\Phi^{\prime \prime}-\mathrm{J}+\mathrm{I}_{2}+\mathrm{I}_{3}+\int_{\Omega}|\nabla u|^{2} d x=\int_{\Omega} \phi_{2}(x,|\nabla u|)|\nabla u|^{2} d x .
$$

Our next goal is to obtain upper estimates for $I_{2}$ and $I_{3}$ by using (3.28) and (3.27). We treat $\mathrm{I}_{2}$ first and exploit the growth condition (3.1). This yields the following chain of inequalities:

$$
\begin{aligned}
\left|\mathrm{I}_{2}\right| & \leq \int_{\Omega}\left|\nabla u^{\prime}\right|\left|\phi_{1}(x,|\nabla u|)\right||\nabla u| d x \leq\left\|\nabla u^{\prime}\right\|_{2}\left(\int_{\Omega}\left|\phi_{1}(x,|\nabla u|)\right|^{2}|\nabla u|^{2} d x\right)^{1 / 2} \\
& \leq \frac{\varepsilon}{2}\left\|\nabla u^{\prime}\right\|_{2}^{2}+\frac{1}{2 \varepsilon} \int_{\Omega}\left|\phi_{1}(x,|\nabla u|)\right|^{2}|\nabla u|^{2} d x \\
& \leq \frac{\varepsilon}{2} \mathrm{~J}+\frac{2 c_{1}^{2}}{2 \varepsilon} \int_{\Omega}|\nabla u|^{2} d x+\frac{2 c_{2}^{2}}{2 \varepsilon} \int_{\Omega}|\nabla u|^{2\left(1+q_{1}\right)} d x \\
& \leq \frac{\varepsilon}{2} \mathrm{~J}+\frac{2 c_{1}^{2}}{\varepsilon} \Phi+\frac{c_{2}^{2}}{\varepsilon} \int_{\Omega}|\nabla u|^{2\left(1+q_{1}\right)} d x,
\end{aligned}
$$

where we have denoted $q_{1}=p_{1}-2$, and $\varepsilon>0$ is strictly positive. Now, we consider the integral

$$
\mathrm{I}_{4}=\int_{\Omega}|\nabla u|^{2\left(1+q_{1}\right)} d x
$$

Since $p_{1} \in(2,4]$ by (ii) $)_{1}$, it follows that $q_{1}=p_{1}-2 \in(0,2]$. Therefore, we have the following continuous embedding:

$$
\mathbb{H}_{0}^{2}(\Omega) \subset \mathbb{W}_{0}^{1, q}(\Omega) \quad \text { for } \quad q=2\left(1+q_{1}\right) .
$$

Denote by $c_{5}>0$ the best possible constant in the inequality

$$
\|\nabla w\|_{q} \leq c_{5}\|\Delta w\|_{2} \quad \text { for all } \quad w \in \mathbb{H}_{0}^{2}(\Omega) .
$$

We have

$$
\begin{aligned}
\mathrm{I}_{4} & =\int_{\Omega}|\nabla u|^{2\left(1+q_{1}\right)} d x \leq c_{5}^{q}\left(\int_{\Omega}|\triangle u|^{2} d x\right)^{q / 2} \\
& =c_{5}^{q} 2^{q / 2}\left(\frac{1}{2} \int_{\Omega}|\triangle u|^{2} d x\right)^{q / 2} \leq c_{5}^{q} 2^{q / 2} \Phi^{1+q_{1}}, \quad q_{1}=p_{1}-2 .
\end{aligned}
$$


Together with (3.30), this yields the following estimate for $\mathrm{I}_{2}$ :

$$
\left|\mathrm{I}_{2}\right| \leq \frac{\varepsilon}{2} \mathrm{~J}+\frac{2 c_{1}^{2}}{\varepsilon} \Phi+\frac{c_{2}^{2} c_{5}^{q} 2^{q / 2}}{\varepsilon} \Phi^{1+q_{1}}, \quad q_{1}=p_{1}-2, \quad \varepsilon>0 .
$$

Now, we estimate $\mathrm{I}_{3}$ by using (3.27). We have

$$
\begin{aligned}
\left|\mathrm{I}_{3}\right| & \leq \int_{\Omega}\left|\nabla u^{\prime}\right|\left|\phi_{1 s}^{\prime}(x,|\nabla u|)\right||\nabla u|^{2} d x \leq\left\|\nabla u^{\prime}\right\|_{2}\left(\int_{\Omega}\left|\phi_{1 s}^{\prime}(x,|\nabla u|)\right|^{2}|\nabla u|^{4} d x\right)^{1 / 2} \\
& \leq \frac{\varepsilon}{2}\left\|\nabla u^{\prime}\right\|_{2}^{2}+\frac{1}{2 \varepsilon} \int_{\Omega}\left|\phi_{1 s}^{\prime}(x,|\nabla u|)\right|^{2}|\nabla u|^{2}|\nabla u|^{2} d x .
\end{aligned}
$$

Observe that the functions $\phi_{1 s}^{\prime}(x, s) s$ and $\phi_{1}(x, s)$ obey similar growth conditions with the same constants (see (ii) $)_{1}$ ). So, we can deduce the same estimate for $I_{3}$ as has been obtained for $\mathrm{I}_{2}$, namely,

$$
\left|\mathrm{I}_{3}\right| \leq \frac{\varepsilon}{2} \mathrm{~J}+\frac{2 c_{1}^{2}}{\varepsilon} \Phi+\frac{c_{2}^{2} c_{5}^{q} 2^{q / 2}}{\varepsilon} \Phi^{1+q_{1}}, \quad q_{1}=p_{1}-2, \quad \varepsilon>0 .
$$

Finally, (3.33), (3.35), and (3.29) imply

$$
\Phi^{\prime \prime}-\mathrm{J}+\varepsilon \mathrm{J}+\frac{4 c_{1}^{2}}{\varepsilon} \Phi+\frac{c_{2}^{2} c_{5}^{q} 2^{q / 2+1}}{\varepsilon} \Phi^{1+q_{1}}+\int_{\Omega}|\nabla u|^{2} d x \geq \int_{\Omega} \phi_{2}(x,|\nabla u|)|\nabla u|^{2} d x
$$

where $q=2\left(1+q_{1}\right)$ and $q_{1}=p_{1}-2$.

We pass to the second energy inequality. For this, consider the functional

$$
\psi(v) \equiv \int_{\Omega} d x \mathcal{F}(x,|\nabla v|), \mathcal{F}(x,|\nabla v|)=\int_{0}^{|\nabla v|} s \phi_{2}(x, s) d s \text { for all } v \in \mathbb{W}_{0}^{1, p_{2}}(\Omega)
$$

It can be rewritten in the form

$$
\psi(h)=\int_{\Omega} d x \mathcal{F}(x, h), \text { where } h=|\nabla v| \in \mathbb{L}^{p_{2}}(\Omega) .
$$

The following lemma is well known.

Lemma 2. Suppose $\phi_{2}(x, s)$ satisfies $(\mathrm{i})_{2}-(\mathrm{ii})_{2}$. Then the functional $\psi(h)$ defined by (3.38) and acting as follows:

$$
\psi(h): \mathbb{L}^{q_{2}+2}(\Omega) \rightarrow \mathbb{R}^{1}, \quad q_{2}=p_{2}-2,
$$

is Fréchet differentiable, with Fréchet derivative given by

$$
\begin{gathered}
\psi_{f}^{\prime}(h): \mathbb{L}^{q_{2}+2}(\Omega) \rightarrow \mathbb{L}^{\left(q_{2}+2\right) /\left(q_{2}+1\right)}(\Omega), \\
\psi_{f}^{\prime}(h)=N_{s \phi_{2}(x, s)}(h) \quad \text { for all } h(x) \in \mathbb{L}^{q_{2}+2}(\Omega) \quad \text { and a.e. } \quad x \in \Omega .
\end{gathered}
$$

We also need the following result (see, e.g., [12]).

Theorem 1. Consider two operators $\mathbb{F}: \mathbb{B}_{1} \rightarrow \mathbb{B}_{2}$ and $\mathbb{G}: \mathbb{B}_{2} \rightarrow \mathbb{B}_{3}$ and suppose that $\mathbb{F}$ is Fréchet differentiable at a point $u \in \mathbb{B}_{1}$ and $\mathbb{G}$ is Fréchet differentiable at $\mathbb{F}(u)$. Then the composition

$$
\mathbb{K} \equiv \mathbb{G} \circ \mathbb{F}
$$

is Fréchet differentiable at $u \in \mathbb{B}_{1}$ and

$$
\mathbb{K}_{f}^{\prime}(u)=\mathbb{G}_{f}^{\prime}(\mathbb{F}(u)) \mathbb{F}_{f}^{\prime}(u) .
$$

By Lemma 2, it follows that for the functional $\psi(u)$ defined by (3.37) we have

$$
\frac{d}{d t} \psi(u)=\int_{\Omega}|\nabla u| \phi_{2}(x,|\nabla u|) \frac{\partial}{\partial t}|\nabla u| d x
$$

whenever $u(x, t) \in \mathbb{C}^{(1)}\left([0, \mathrm{~T}] ; \mathbb{H}_{0}^{2}(\Omega)\right)$. 
We do elementary transformations in (3.40):

$$
\begin{aligned}
\int_{\Omega} \mid & \left.\nabla u\left|\phi_{2}(x,|\nabla u|) \frac{\partial}{\partial t}\right| \nabla u\left|d x=\frac{1}{2} \int_{\Omega} \phi_{2}(x,|\nabla u|) \frac{\partial}{\partial t}\right| \nabla u\right|^{2} d x \\
= & \int_{\Omega} \phi_{2}(x,|\nabla u|)\left(\nabla u^{\prime}, \nabla u\right) d x=\int_{\Omega}\left(\nabla u^{\prime}, \phi_{2}(x,|\nabla u|) \nabla u\right) d x \\
= & -\left\langle\operatorname{div}\left(\phi_{2}(x,|\nabla u|) \nabla u\right), u^{\prime}\right\rangle_{2}=-\left\langle\operatorname{div}\left(\phi_{2}(x,|\nabla u|) \nabla u\right), u^{\prime}\right\rangle ;
\end{aligned}
$$

we recall that $\langle\cdot, \cdot\rangle_{2}$ stands here for the pairing between $\mathbb{W}_{0}^{1, p_{2}}(\Omega)$ and $\mathbb{W}^{-1, p_{2}^{\prime}}(\Omega)$. The last identity is true by (3.18).

Now, we pass directly to the second energy inequality. For the role of $w$ in (3.19), we take the time derivative of the solution: $w=u^{\prime}$. After "integration by parts", taking into account (3.41) and the definition (3.21) of J, we arrive at

$$
\begin{array}{r}
\frac{1}{2} \frac{d}{d t} \mathrm{~J}+\int_{\Omega}\left(\nabla u^{\prime}, \frac{\partial}{\partial t}\left[\phi_{1}(x,|\nabla u|) \nabla u\right]\right) d x+\frac{1}{2} \frac{d}{d t} \int_{\Omega}|\nabla u|^{2} d x \\
=-\left\langle\operatorname{div}\left(\phi_{2}(x,|\nabla u|) \nabla u\right), u^{\prime}\right\rangle=\frac{d}{d t} \int_{\Omega} \mathcal{F}(x,|\nabla u|) d x .
\end{array}
$$

Consider the following integral separately:

$$
\begin{aligned}
\mathrm{I}_{5} & \equiv \int_{\Omega}\left(\nabla u^{\prime}, \frac{\partial}{\partial t}\left[\phi_{1}(x,|\nabla u|) \nabla u\right]\right) d x \\
& =\int_{\Omega}\left(\nabla u^{\prime}, \nabla u^{\prime}\right) \phi_{1}(x,|\nabla u|) d x+\left.\int_{\Omega}\left(\nabla u^{\prime}, \nabla u\right) \phi_{1 s}^{\prime}(x, s)\right|_{s=|\nabla u|} \frac{\partial}{\partial t}|\nabla u| d x \\
& =\int_{\Omega} \phi_{1}(x,|\nabla u|)\left|\nabla u^{\prime}\right|^{2} d x+\left.\int_{\Omega} \phi_{1 s}^{\prime}(x, s)\right|_{s=|\nabla u|} \frac{\partial}{\partial t}|\nabla u| \frac{1}{2} \frac{\partial}{\partial t}|\nabla u|^{2} d x \\
& =\int_{\Omega} \phi_{1}(x,|\nabla u|)\left|\nabla u^{\prime}\right|^{2} d x+\left.\int_{\Omega} \phi_{1 s}^{\prime}(x, s)\right|_{s=|\nabla u|}|\nabla u|\left[\frac{\partial}{\partial t}|\nabla u|\right]^{2} d x \geq 0,
\end{aligned}
$$

where the last inequality holds true by (3.3). Together with (3.42), this shows that

$$
\begin{aligned}
\frac{1}{2} \frac{d}{d t} \mathrm{~J}+\frac{1}{2} \frac{d}{d t} \int_{\Omega}|\nabla u|^{2} d x & \leq \frac{d}{d t} \int_{\Omega} \mathcal{F}(x,|\nabla u|) d x \\
\mathcal{F}(x,|\nabla u|) & =\int_{0}^{|\nabla u|} s \phi_{2}(x, s) d s .
\end{aligned}
$$

We introduce the following notation:

$$
\mathrm{E}(t) \equiv \int_{\Omega} \mathcal{F}(x,|\nabla u|) d x-\frac{1}{2} \mathrm{~J}(t)-\frac{1}{2} \int_{\Omega}|\nabla u|^{2} d x .
$$

Clearly,

$$
\mathrm{E}(t) \geq \mathrm{E}(0) .
$$

Now, we impose the first condition on the initial data:

$$
\mathrm{E}(0)=\int_{\Omega} \mathcal{F}\left(x,\left|\nabla u_{0}\right|\right) d x-\frac{1}{2} \int_{\Omega}\left|\triangle u_{1}\right|^{2} d x-\frac{1}{2} \int_{\Omega}\left|\nabla u_{1}\right|^{2} d x-\frac{1}{2} \int_{\Omega}\left|\nabla u_{0}\right|^{2} d x \geq 0 .
$$

Together with (3.45), this yields

$$
\mathrm{E}(t) \geq 0 \text { for all } t \in[0, \mathrm{~T}] .
$$

Using property (iii) $)_{2}$ for $\phi_{2}(x, s)$ and (3.47), we deduce the inequality

$$
\int_{\Omega}|\nabla u|^{2} \phi_{2}(x,|\nabla u|) d x \geq \frac{\vartheta}{2} \mathrm{~J}(t)+\frac{\vartheta}{2} \int_{\Omega}|\nabla u|^{2} d x .
$$


Combining this with (3.36), we obtain

$$
\begin{aligned}
\Phi^{\prime \prime}-\left(1+\frac{\vartheta}{2}\right) \mathrm{J} & +\varepsilon \mathrm{J}+\frac{4 c_{1}^{2}}{\varepsilon} \Phi+\frac{c_{2}^{2} c_{5}^{q} 2^{q / 2+1}}{\varepsilon} \Phi^{1+q_{1}}+\left(1-\frac{\vartheta}{2}\right) \int_{\Omega}|\nabla u|^{2} d x \\
& \geq \int_{\Omega}|\nabla u|^{2} \phi_{2}(x,|\nabla u|) d x-\frac{\vartheta}{2} \mathrm{~J}(t)-\frac{\vartheta}{2} \int_{\Omega}|\nabla u|^{2} d x \geq 0 .
\end{aligned}
$$

Since $\vartheta>2$, it follows that

$$
\begin{gathered}
\left(1+\frac{\vartheta}{2}-\varepsilon\right) \mathrm{J} \leq \Phi^{\prime \prime}+\frac{c_{7}}{\varepsilon} \Phi+\frac{c_{6}}{\varepsilon} \Phi^{1+q_{1}}, \quad q_{1}=p_{1}-2, \\
c_{7}=4 c_{1}^{2}, \quad c_{6}=c_{2}^{2} c_{5}^{q} 2^{q / 2+1}, \quad q=2\left(q_{1}+1\right),
\end{gathered}
$$

provided

$$
\frac{1}{2}\left(1+\frac{\vartheta}{2}-\varepsilon\right)>1 \Rightarrow \varepsilon \in\left(0, \frac{\vartheta-2}{2}\right)
$$

Inequalities (3.49) and (3.22) lead to the following first order ordinary differential inequality:

$$
\Phi \Phi^{\prime \prime}-\alpha\left(\Phi^{\prime}\right)^{2}+\beta \Phi^{2}+\gamma \Phi^{2+q_{1}} \geq 0, \quad q_{1}=p_{1}-2
$$

where

$$
\alpha=\frac{1}{2}\left(1+\frac{\vartheta}{2}-\varepsilon\right), \quad \beta=\frac{c_{7}}{\varepsilon}, \quad \gamma=\frac{c_{6}}{\varepsilon}, \quad \varepsilon \in\left(0, \frac{\vartheta-2}{2}\right), \quad \vartheta>2 .
$$

We introduce the new function

$$
\Psi=\Phi^{1-\alpha} .
$$

Dividing the two sides of (3.50) by $\Phi^{1+\alpha} \geq 0$ (see the definition (3.20) ), we arrive at the inequality

$$
\begin{aligned}
& \frac{\Phi^{\prime \prime}}{\Phi^{\alpha}}-\frac{\alpha\left(\Phi^{\prime}\right)^{2}}{\Phi^{1+\alpha}}+\beta \Phi^{1-\alpha}+\gamma \Phi^{1+q_{1}-\alpha} \geq 0 \\
& \quad \Rightarrow \frac{d}{d t}\left[\frac{\Phi^{\prime}}{\Phi^{\alpha}}\right]+\beta \Phi^{1-\alpha}+\gamma \Phi^{1+q_{1}-\alpha} \geq 0 \\
& \quad \Rightarrow \frac{1}{1-\alpha} \frac{d^{2}}{d t^{2}} \Phi^{1-\alpha}+\beta \Phi^{1-\alpha}+\gamma \Phi^{1+q_{1}-\alpha} \geq 0 .
\end{aligned}
$$

Invoking the notation (3.51), we obtain

$$
\frac{1}{1-\alpha} \Psi^{\prime \prime}+\beta \Psi+\gamma \Psi^{\left(1+q_{1}-\alpha\right) /(1-\alpha)} \geq 0 .
$$

Now, we impose another assumption on the initial data:

$$
\Phi^{\prime}(0)=\int_{\Omega}\left(\nabla u_{1}, \nabla u_{0}\right) d x+\int_{\Omega} \triangle u_{1} \triangle u_{0} d x>0 .
$$

Since $u(x, t) \in \mathbb{C}^{(2)}\left([0, T] ; \mathbb{H}_{0}^{2}(\Omega)\right)$ by assumption, we have $\Phi(t) \in \mathbb{C}^{(1)}([0, T])$. Consequently, there is some moment $t_{1}>0$ with

$$
\Phi^{\prime}(t)>0 \text { for all } t \in\left[0, t_{1}\right] .
$$

Observe that by (3.51) we have

$$
\Psi^{\prime}=(1-\alpha) \Phi^{-\alpha} \Phi^{\prime}
$$

Since $\alpha>1$, (3.54) shows that

$$
\Psi^{\prime}(t) \leq 0 \quad \text { for all } t \in\left[0, t_{1}\right] .
$$


Then, multiplying (3.52) by $\Psi^{\prime}$, we arrive at

$$
\frac{1}{1-\alpha} \Psi^{\prime} \Psi^{\prime \prime}+\beta \Psi^{\prime} \Psi+\gamma \Psi^{\prime} \Psi^{\left(1+q_{1}-\alpha\right) /(1-\alpha)} \leq 0 \quad \text { for all } t \in\left[0, t_{1}\right],
$$

whence it follows that

$$
\Psi^{\prime} \Psi^{\prime \prime} \geq \beta(\alpha-1) \Psi^{\prime} \Psi+\gamma(\alpha-1) \Psi^{\prime} \Psi^{\left(1+q_{1}-\alpha\right) /(1-\alpha)} \quad \text { for all } \quad t \in\left[0, t_{1}\right] .
$$

This implies the inequality

$$
\frac{1}{2} \frac{d}{d t}\left(\Psi^{\prime}\right)^{2} \geq \frac{\beta(\alpha-1)}{2} \frac{d}{d t} \Psi^{2}+\frac{\gamma(\alpha-1)}{\delta} \frac{d}{d t} \Psi^{\delta}
$$

where

We need to guarantee that $\delta>0$. Observe that

$$
\delta=1+\frac{1+q_{1}-\alpha}{1-\alpha} .
$$

$$
\begin{aligned}
\delta>0 & \Rightarrow \frac{1-\alpha+1+q_{1}-\alpha}{1-\alpha}>0 \Rightarrow \frac{2 \alpha-2-q_{1}}{\alpha-1}>0 \Rightarrow 2 \alpha>q_{1}+2 \\
& \Rightarrow 1+\frac{\vartheta}{2}-\varepsilon>q_{1}+2 \Rightarrow \varepsilon \in\left(0, \frac{\vartheta-2 q_{1}-2}{2}\right) .
\end{aligned}
$$

Thus, we must impose yet another condition on the quantity $\vartheta>2$ in (iii) $)_{2}$, specifically,

$$
\vartheta>2+2 q_{1}, \quad q_{1}=p_{1}-2 .
$$

Remark 3. Note that functions $\phi_{2}(x,|\nabla u|)$ obeying (3.58) do exist. Indeed, let

$$
\phi_{2}(x,|\nabla u|)=|\nabla u|^{p_{2}-2} \text { for } q_{2}>2 q_{1},
$$

where $q_{1}=p_{1}-2$ and $q_{2}=p_{2}-2$. Then $\vartheta=q_{2}+2$ for this function, and (3.58) is fulfilled if $q_{2}>2 q_{1}$.

Now, let (3.58) be true. We continue the analysis of inequality (3.57). After integration, it implies

$$
\left(\Psi^{\prime}\right)^{2} \geq A^{2}+\beta(\alpha-1) \Psi^{2}+\frac{2 \gamma(\alpha-1)}{\delta} \Psi^{\delta} \geq A^{2}
$$

where

$$
A^{2} \equiv\left(\Psi^{\prime}(0)\right)^{2}-\beta(\alpha-1) \Psi^{2}(0)-\frac{2 \gamma(\alpha-1)}{\delta} \Psi^{\delta}(0) .
$$

We subject the initial data to yet another condition, namely,

$$
A^{2}>0 \text {. }
$$

Taking the definition (3.51) of $\Psi(t)$ into account, from (3.61) we obtain the following equivalent inequality:

$$
(\alpha-1)^{2} \Phi_{0}^{-2 \alpha}\left(\Phi^{\prime}(0)\right)^{2}-\beta(\alpha-1) \Phi_{0}^{-2 \alpha} \Phi_{0}^{2}-\frac{2 \gamma(\alpha-1)}{\delta} \Phi_{0}^{-\delta \alpha} \Phi_{0}^{\delta}>0,
$$

which implies

$$
\left(\Phi^{\prime}(0)\right)^{2}>\frac{\beta}{\alpha-1} \Phi_{0}^{2}+\frac{2 \gamma}{(\alpha-1) \delta} \Phi_{0}^{\alpha(2-\delta)+\delta}
$$

Next, clearly,

$$
\begin{aligned}
\alpha(2-\delta)+\delta & =2 \alpha+(1-\alpha) \delta=2 \alpha+1-\alpha+1+q_{1}-\alpha=2+q_{1}, \\
\delta(\alpha-1) & =\alpha-1-1-q_{1}+\alpha=2 \alpha-q_{1}-2 .
\end{aligned}
$$

So, (3.62) yields

$$
\left(\Phi^{\prime}(0)\right)^{2}>\frac{\beta}{\alpha-1} \Phi_{0}^{2}+\frac{2 \gamma}{2 \alpha-q_{1}-2} \Phi_{0}^{2+q_{1}}, \quad q_{1}=p_{1}-2,
$$


where $2 \alpha-q_{1}-2>0$ provided that $\vartheta>2+2 q_{1}$ and

$$
\varepsilon \in\left(0, \frac{\vartheta-2 q_{1}-2}{2}\right) .
$$

Remark 4. Our immediate task is to show that the set of functions $\phi_{1}(x, s)$ and $\phi_{2}(x, s)$ and the initial data $u_{0}(x), u_{1}(x)$ that satisfy (3.46), (3.53), and (3.63) is nonempty. Indeed, let $2 q_{1}<q_{2}\left(q_{1}=p_{1}-2\right.$ and $\left.q_{2}=p_{2}-2\right)$ and, moreover, let $q_{3} \in\left(2 q_{1}, q_{2}\right)$. We fix $u_{0}(x) \in \mathbb{C}_{0}^{\infty}(\Omega) \subset \mathbb{H}_{0}^{2}(\Omega)$ (recall that $\partial \Omega \in \mathbb{C}^{4, \delta}$ with $\delta \in(0,1]$ ) such that $\left\|\triangle u_{0}\right\|_{2}>0$ and consider the classical solution $u_{1}(x) \in \mathbb{C}^{4+\mu}(\bar{\Omega}) \cap \mathbb{C}_{0}^{(2)}(\bar{\Omega})$ of the following problem:

$$
\begin{aligned}
\triangle^{2} u_{1}(x)-\triangle u_{1}(x) & =\left(\left|u_{0}\right|^{q_{3} / 2} u_{0}\right)(x) \in \mathbb{C}^{\mu}(\bar{\Omega}), \quad \mu=\mu\left(q_{3}\right) \in(0,1], \\
\left.u_{1}\right|_{\partial \Omega} & =\left.\frac{\partial u_{1}}{\partial n_{x}}\right|_{\partial \Omega}=0 .
\end{aligned}
$$

Then (3.53) can be transformed to

$$
\begin{aligned}
\Phi^{\prime}(0) & =\int_{\Omega} \triangle u_{1} \triangle u_{0} d x+\int_{\Omega}\left(\nabla u_{1}, \nabla u_{0}\right) d x \\
& =\int_{\Omega}\left[\triangle^{2} u_{1}-\triangle u_{1}\right] u_{0} d x=\int_{\Omega}\left|u_{0}\right|^{2+q_{3} / 2} d x>0,
\end{aligned}
$$

which is clearly true. Now, we verify (3.63). After a substitution, we arrive at the inequality

$$
\left(\int_{\Omega}\left|u_{0}\right|^{2+q_{3} / 2} d x\right)^{2}>\frac{\beta}{\alpha-1} \Phi_{0}^{2}+\frac{2 \gamma}{2 \alpha-q_{1}-2} \Phi_{0}^{2+q_{1}}
$$

Now in place of $u_{0}(x)$ we put $r u_{0}(x)$, where $r>0$. Then (3.53) does not change, while the last inequality takes the form

$$
r^{4+q_{3}}\left(\int_{\Omega}\left|u_{0}\right|^{2+q_{3} / 2} d x\right)^{2}>r^{4} \frac{\beta}{\alpha-1} \Phi_{0}^{2}+r^{4+2 q_{1}} \frac{2 \gamma}{2 \alpha-q_{1}-2} \Phi_{0}^{2+q_{1}} .
$$

Since $q_{3}>2 q_{1}$, it follows that (3.44) is fulfilled for sufficiently large $r>0$. Finally, we analyze condition (3.47); for convenience, we reproduce it:

$$
\int_{\Omega} \mathcal{F}\left(x,\left|\nabla u_{0}\right|\right) d x \geq \frac{1}{2} \int_{\Omega}\left|\nabla u_{1}\right|^{2} d x+\frac{1}{2} \int_{\Omega}\left|\triangle u_{1}\right|^{2} d x+\frac{1}{2} \int_{\Omega}\left|\nabla u_{0}\right|^{2} d x .
$$

We put $\phi_{2}(x,|\nabla u|)=|\nabla u|^{q_{2}}$ with $q_{2}=p_{2}-2$. Then after integration we obtain

$$
\int_{\Omega} \mathcal{F}\left(x,\left|\nabla u_{0}\right|\right) d x=\frac{1}{q_{2}+2} \int_{\Omega}\left|\nabla u_{0}\right|^{q_{2}+2} d x .
$$

This yields the inequality

$$
\frac{1}{2} \int_{\Omega}\left|\nabla u_{1}\right|^{2} d x+\frac{1}{2} \int_{\Omega}\left|\triangle u_{1}\right|^{2} d x+\frac{1}{2} \int_{\Omega}\left|\nabla u_{0}\right|^{2} d x \leq \frac{1}{q_{2}+2} \int_{\Omega}\left|\nabla u_{0}\right|^{q_{2}+2} d x .
$$

Now we invoke the Green function $\mathrm{K}(x, y)$ for the linear nonhomogeneous problem (3.64). The Green function in question exists by the results of [5] (the corresponding Green operator acts as follows: $\left.\mathbb{K}: \mathbb{C}^{\mu}(\bar{\Omega}) \rightarrow \mathbb{C}^{4+\mu}(\bar{\Omega})\right)$. Then we have the following formula for $u_{1}(x)$ :

$$
u_{1}(x)=\int_{\Omega} \mathrm{K}(x, y)\left(\left|u_{0}\right|^{q_{3} / 2} u_{0}\right)(y) d y
$$


Substituting this in (3.65) and replacing $u_{0}$ with $r u_{0}$, we obtain the inequality

$$
\begin{aligned}
& r^{2+q_{3}} \frac{1}{2} \int_{\Omega}\left|\int_{\Omega} \nabla_{x} \mathrm{~K}(x, y)\left(\left|u_{0}\right|^{q_{3} / 2} u_{0}\right)(y) d y\right|^{2} d x \\
& \quad+r^{2+q_{3}} \frac{1}{2} \int_{\Omega}\left|\int_{\Omega} \triangle_{x} \mathrm{~K}(x, y)\left(\left|u_{0}\right|^{q_{3} / 2} u_{0}\right)(y) d y\right|^{2} d x+r^{2} \frac{1}{2} \int_{\Omega}\left|\nabla u_{0}\right|^{2} d x \\
& \quad \leq r^{q_{2}+2} \frac{1}{q_{2}+2} \int_{\Omega}\left|\nabla u_{0}\right|^{q_{2}+2} d x .
\end{aligned}
$$

Clearly, this inequality is fulfilled for sufficiently large $r>0$, because $q_{3}<q_{2}$. Thus, conditions (3.46), (3.53), and (3.63) are compatible.

We continue the study of (3.59). This inequality implies

$$
\left|\Psi^{\prime}\right| \geq A>0 \quad \text { for } \quad t \in\left[0, t_{1}\right]
$$

Consequently,

$$
-\Psi^{\prime} \geq A>0 \Rightarrow \Psi^{\prime} \leq-A<0 \text { for all } t \in\left[0, t_{1}\right] .
$$

Now we recall that

$$
\Psi^{\prime}(t)=(1-\alpha) \Phi^{-\alpha} \Phi^{\prime}(t)
$$

Therefore,

$$
(1-\alpha) \Phi^{-\alpha} \Phi^{\prime}(t) \leq-A \Rightarrow \Phi^{\prime} \geq \frac{A}{\alpha-1} \Phi^{\alpha}>0
$$

but this implies that, under condition (3.53), the quantity $\Phi^{\prime}(t)$ remains strictly positive for the entire interval where the solution of the problem in question exists. Thus, (3.66) shows that

$$
\Psi^{\prime}(t) \leq-A<0 \text { for all } t \in\left[0, \mathrm{~T}_{0}\right),
$$

where $\mathrm{T}_{0}$ is the time of existence for the solution. Integrating the last inequality, we arrive at

$$
\Psi(t) \leq \Psi_{0}-A t
$$

therefore, after a finite time $\mathrm{T}_{0} \in\left[0, \mathrm{~T}_{1}\right]$, the function $\Psi(t)$ acquires a zero; here

$$
\mathrm{T}_{1}=\frac{\Phi_{0}^{1-\alpha}}{A}>0
$$

Consequently,

$$
\limsup _{t \uparrow T_{0}} \Phi(t)=+\infty
$$

Now, we must choose the parameter

$$
\varepsilon \in\left(0, \frac{\vartheta-2 q_{1}-2}{2}\right)
$$

in an optimal way. We choose

$$
\varepsilon_{0} \in\left(0, \frac{\vartheta-2 q_{1}-2}{2}\right)
$$

so that the quantity

$$
Q_{0}=Q_{0}(\varepsilon) \equiv \frac{\beta}{\alpha-1}+\frac{2 \gamma}{2 \alpha-q_{1}-2} \Phi_{0}^{q_{1}}, \quad q_{1}=p_{1}-2,
$$

be the smallest possible. Clearly, $\varepsilon_{0}$ is a function of $\Phi_{0}$.

So, we have obtained the following result: under conditions (3.46), (3.53), (3.58), and (3.63), the strong generalized solution $u(x, t) \in \mathbb{C}^{(2)}\left([0, \mathrm{~T}] ; \mathbb{H}_{0}^{2}(\Omega)\right)$ of problem (2.11)(2.13) in the sense of Definition 3 cannot exist globally in time. Now we want to show 
that $\mathrm{T}_{0}=\mathrm{T}_{0}\left(u_{0}, u_{1}\right)>0$, i.e., the problem is solvable locally in time in the strong generalized sense whenever $u_{0}, u_{1} \in \mathbb{H}_{0}^{2}(\Omega)$. This will be done in the next section.

\section{$\S 4$. Local SOLVABiLity}

Problem (2.11)-(2.13) can be rewritten in the following form:

$$
\begin{aligned}
& \frac{d^{2}}{d t^{2}} \mathbb{A} u+\frac{d}{d t} \mathbb{B}_{1}(u)+\mathbb{L} u=\mathbb{B}_{2}(u), \\
& u(0)=u_{0}, \quad u^{\prime}(0)=u_{1}, \quad u_{0}(x), u_{1}(x) \in \mathbb{H}_{0}^{2}(\Omega),
\end{aligned}
$$

where

$$
\begin{aligned}
& \mathbb{A} \equiv \triangle^{2}-\triangle, \quad \mathbb{B}_{1}(u) \equiv-\operatorname{div}\left(\phi_{1}(x,|\nabla u|) \nabla u\right), \\
& \mathbb{B}_{2}(u) \equiv-\operatorname{div}\left(\phi_{2}(x,|\nabla u|) \nabla u\right), \quad \mathbb{L} \equiv-\triangle .
\end{aligned}
$$

We need to know that the operator

$$
\mathbb{A} \equiv \triangle^{2}-\triangle: \mathbb{H}_{0}^{2}(\Omega) \rightarrow \mathbb{H}^{-2}(\Omega)
$$

is invertible, and that the inverse

$$
\mathbb{A}^{-1}: \mathbb{H}^{-2}(\Omega) \rightarrow \mathbb{H}_{0}^{2}(\Omega)
$$

is Lipschitz continuous. To prove this, we shall use the following version of the BrowderMinty theorem (see, e.g., [1]).

Browder-Minty theorem. Suppose that an operator $\mathbb{A}$ acting from a Banach space $\mathbb{W}$ to the strong dual $\mathbb{W}^{*}$ is radially continuous, strongly monotone, and coercive. Then $\mathbb{A}$ admits a Lipschitz continuous inverse $\mathbb{A}^{-1}: \mathbb{W}^{*} \rightarrow \mathbb{W}$.

Three notions are involved in this theorem. For completeness, we give the definitions.

Definition 4. An operator $\mathbb{A}: \mathbb{W} \rightarrow \mathbb{W}^{*}$ is said to be radially continuous if for all $u$, $v \in \mathbb{W}$ the function

$$
\phi(s)=\langle\mathbb{A}(u+s v), v\rangle
$$

belongs to $\mathbb{C}[0,1]$, where $\langle\cdot, \cdot\rangle$ denotes the pairing between $\mathbb{W}$ and $\mathbb{W}^{*}$.

Definition 5. An operator $\mathbb{A}: \mathbb{W} \rightarrow \mathbb{W}^{*}$ is said to be strongly monotone if there is a constant $m>0$ such that

$$
\langle\mathbb{A}(u)-\mathbb{A}(v), u-v\rangle \geq m\|u-v\|^{2},
$$

where $\|\cdot\|$ is the norm in $\mathbb{W}$.

Definition 6. An operator $\mathbb{A}: \mathbb{W} \rightarrow \mathbb{W}^{*}$ is said to be coercive if there exists a function

$$
\gamma(s): \mathbb{R}_{+}^{1} \rightarrow \mathbb{R}_{+}^{1} \quad \lim _{s \rightarrow+\infty} \gamma(s)=+\infty
$$

such that

$$
\langle\mathbb{A}(u), u\rangle \geq \gamma(\|u\|)\|u\| \quad \text { for all } u \in \mathbb{W} .
$$

Now we must show that the operator $\mathbb{A}$ defined by (4.2) is radially continuous, strongly monotone and coercive as a mapping from $\mathbb{W}=\mathbb{H}_{0}^{2}(\Omega)$ to $\mathbb{W}^{*}=\mathbb{H}^{-2}(\Omega)$, that is, it satisfies the assumptions of the Browder-Minty theorem.

(I) We show that $\mathbb{A}$ is radially continuous. Indeed, consider the expression

$$
\begin{aligned}
\phi(s) & =\langle\mathbb{A}(u+s v), v\rangle=\int_{\Omega}(\nabla(u+s v), \nabla v) d x+\int_{\Omega}(\triangle u+s \triangle v) \triangle v d x \\
& =\int_{\Omega}(\nabla u, \nabla v) d x+s \int_{\Omega}|\nabla v|^{2} d x+\int_{\Omega} \triangle u \triangle v d x+s \int_{\Omega}|\triangle v|^{2} d x .
\end{aligned}
$$


Clearly, $\phi(s) \in \mathbb{C}[0,1]$ for every $u, v \in \mathbb{H}_{0}^{2}(\Omega)$.

Hence, $\mathbb{A}: \mathbb{H}_{0}^{2}(\Omega) \rightarrow \mathbb{H}^{-2}(\Omega)$ is radially continuous.

(II) Next, we show that $\mathbb{A}$ is strongly monotone. Indeed, we have

$$
\begin{aligned}
\langle\mathbb{A}(u)-\mathbb{A}(v), u-v\rangle & =\int_{\Omega}|\nabla u-\nabla v|^{2} d x+\int_{\Omega}|\Delta u-\triangle v|^{2} d x \\
& \geq \int_{\Omega}|\triangle u-\Delta v|^{2} d x=\|u-v\|^{2},
\end{aligned}
$$

where $\|\cdot\|$ stands for the norm in $\mathbb{H}_{0}^{2}(\Omega)$. Thus, $\mathbb{A}: \mathbb{H}_{0}^{2}(\Omega) \rightarrow \mathbb{H}^{-2}(\Omega)$ is strongly monotone with constant $m=1$.

(III) Finally, we prove that $\mathbb{A}$ is coercive. Indeed,

$$
\langle\mathbb{A}(u), u\rangle=\int_{\Omega}|\nabla u|^{2} d x+\int_{\Omega}|\triangle u|^{2} d x \geq \int_{\Omega}|\triangle u|^{2} d x=\|u\|^{2} .
$$

Thus, $\mathbb{A}$ is coercive with $\gamma(s)=s$.

Now, the Browder-Minty theorem is applicable to $\mathbb{A}: \mathbb{H}_{0}^{2}(\Omega) \rightarrow \mathbb{H}^{-2}(\Omega)$ (defined by (4.2)), yielding the statement that this operator admits a Lipschitz continuous inverse

$$
\mathbb{A}^{-1}: \mathbb{H}^{-2}(\Omega) \rightarrow \mathbb{H}_{0}^{2}(\Omega) .
$$

We show that, moreover, the Lipschitz constant is equal to 1. By (II), we have

$$
\langle\mathbb{A}(u)-\mathbb{A}(v), u-v\rangle \geq\|u-v\|^{2} \quad \text { for all } \quad u, v \in \mathbb{H}_{0}^{1}(\Omega)
$$

Since the norm $\|\cdot\|_{*}$ of the strong dual $\mathbb{H}^{-2}(\Omega)$ to $\mathbb{H}_{0}^{2}(\Omega)$ is given standardly by

$$
\|f\|_{*} \equiv \sup _{\substack{v \in \mathbb{H}_{0}^{2}(\Omega),\|v\| \leq 1}}|\langle f, v\rangle|,
$$

we obtain

$$
\|\mathbb{A}(u)-\mathbb{A}(v)\|_{*}\|u-v\| \geq\langle\mathbb{A}(u)-\mathbb{A}(v), u-v\rangle .
$$

Indeed, (4.8) follows easily from (4.7), moreover, we have the general inequality

$$
|\langle f, w\rangle| \leq\|f\|_{*}\|w\| .
$$

To prove (4.9), we observe that it is true if $w=\vartheta$, the zero of $\mathbb{H}_{0}^{2}(\Omega)$; but if $w \neq \vartheta$, then

$$
|\langle f, v\rangle| \leq\|f\|_{*} \text { for all }\|v\| \leq 1
$$

by (4.7). In particular, putting

we arrive at

$$
v=\frac{w}{\|w\|}
$$

$$
|\langle f, w\rangle| \leq\|f\|_{*}\|w\| .
$$

Now, (4.8) is a consequence of (4.9) if we put

$$
f=\mathbb{A}(u)-\mathbb{A}(v), \quad w=u-v
$$

in it. By (4.6) and (4.8), we see that

$$
\|u-v\| \leq\|\mathbb{A}(u)-\mathbb{A}(v)\|_{*} \quad \text { for all } \quad u, v \in \mathbb{H}_{0}^{2}(\Omega) .
$$

Now, we put

$$
w_{1}=\mathbb{A}(u), \quad w_{2}=\mathbb{A}(v)
$$

then, by (4.10) and the invertibility of $\mathbb{A}: \mathbb{H}_{0}^{2}(\Omega) \rightarrow \mathbb{H}^{-2}(\Omega)$ proved above, we arrive at the inequality

$$
\left\|\mathbb{A}^{-1}\left(w_{1}\right)-\mathbb{A}^{-1}\left(w_{2}\right)\right\| \leq\left\|w_{1}-w_{2}\right\|_{*} \quad \text { for } \quad w_{1}, w_{2} \in \mathbb{H}^{-2}(\Omega) .
$$


So, we have proved that the operator $\mathbb{A}^{-1}: \mathbb{H}^{-2}(\Omega) \rightarrow \mathbb{H}_{0}^{2}(\Omega)$ is Lipschitz continuous with constant 1.

Since $\mathbb{A}$ is invertible, for $u(x, t) \in \mathbb{C}^{(2)}\left([0, T] ; \mathbb{H}_{0}^{2}(\Omega)\right)$ we can introduce a new function $v=\mathbb{A} u$ and rewrite problem (4.1) in the following equivalent form:

$$
\begin{aligned}
& \frac{d^{2} v}{d t^{2}}+\frac{d}{d t} \mathbb{B}_{1}\left(\mathbb{A}^{-1} v\right)+\mathbb{L} \mathbb{A}^{-1} v=\mathbb{B}_{2}\left(\mathbb{A}^{-1} v\right), \\
& v_{0}=v(0)=\mathbb{A} u_{0} \in \mathbb{H}^{-2}(\Omega), \quad v_{1}=v^{\prime}(0)=\mathbb{A} u_{1} \in \mathbb{H}^{-2}(\Omega) .
\end{aligned}
$$

In the class $v(x, t) \in \mathbb{C}^{(2)}\left([0, \mathrm{~T}] ; \mathbb{H}^{-2}(\Omega)\right)$ for some $\mathrm{T}>0$, problem (4.12) is equivalent to the integral equation

$$
\begin{gathered}
v(t)=w_{1}+\int_{0}^{t} d s G(v)(s), \quad w_{1}=v_{0}+\mathbb{B}_{1}\left(\mathbb{A}^{-1} v_{0}\right) t \\
G(v)(s) \equiv v_{1}-\mathbb{B}_{1}\left(\mathbb{A}^{-1} v(s)\right)+\int_{0}^{s} d \sigma\left[-\mathbb{L} \mathbb{A}^{-1} v(\sigma)+\mathbb{B}_{2}\left(\mathbb{A}^{-1} v(\sigma)\right)\right] .
\end{gathered}
$$

Remark 5. When deducing the integral equation (4.13), we have used the statement that $\mathbb{B}_{1}(w)$ is a continuous operator. This is true indeed: below we shall deduce this from property (iii) $)_{1}$.

We look for a solution of the integral equation (4.13) in the Banach space

$$
\mathbb{L}^{\infty}\left(0, \mathrm{~T} ; \mathbb{H}^{-2}(\Omega)\right) \text {. }
$$

We shall need the following bounded closed convex subset of this Banach space:

$$
\mathbb{V}_{r} \equiv\left\{v \in \mathbb{L}^{\infty}\left(0, \mathrm{~T} ; \mathbb{H}^{-2}(\Omega)\right):\|v\|=\underset{t \in[0, \mathrm{~T}]}{\operatorname{ess} \sup }\|v\|_{*} \leq r\right\} \text { for some } r>0,
$$

where $\|\cdot\|_{*}$ is the norm of the Banach space $\mathbb{H}^{-2}(\Omega)$.

First, we need upper estimates for the operators

$$
\mathbb{B}_{1}\left(\mathbb{A}^{-1} v\right) \text { and } \quad \mathbb{B}_{2}\left(\mathbb{A}^{-1} v\right)
$$

for $v \in \mathbb{V}_{r}$ in the norm $\|\cdot\|_{*}$. For the first operator, by the growth condition (ii) $)_{1}$, we can write

$$
\begin{aligned}
\left\|\mathbb{B}_{1}(w)\right\|_{*} & \leq c_{8}\left\||\nabla w| \phi_{1}(x,|\nabla w|)\right\|_{p_{1}^{\prime}} \leq c_{8}\left[c_{1}\|\nabla w\|_{p_{1}^{\prime}}+c_{2}\left\||\nabla w|^{p_{1}-1}\right\|_{p_{1}^{\prime}}\right] \\
& \leq c_{9}\|\nabla w\|_{p_{1}}+c_{10}\|\nabla w\|_{p_{1}}^{p_{1}-1} \\
& \leq \frac{p_{1}-2}{p_{1}-1} c_{9}^{\left(p_{1}-1\right) /\left(p_{1}-2\right)}+\frac{1}{p_{1}-1}\|\nabla w\|_{p_{1}}^{p_{1}-1}+c_{10}\|\nabla w\|_{p_{1}}^{p_{1}-1} \\
& =c_{11}+c_{12}\|\nabla w\|_{p_{1}}^{p_{1}-1},
\end{aligned}
$$

where $\|\cdot\|_{r}$ stands for the norm in $\mathbb{L}^{r}(\Omega)$. In the last inequality, we have used the fact that $p_{1}>2$ by (ii) ${ }_{1}$. We put $w=\mathbb{A}^{-1} v \in \mathbb{H}_{0}^{2}(\Omega)$. Then (4.16) yields

$$
\left\|\mathbb{B}_{1}\left(\mathbb{A}^{-1} v\right)\right\|_{*} \leq c_{11}+c_{12}\left\|\nabla \mathbb{A}^{-1} v\right\|_{p_{1}}^{p_{1}-1} \leq c_{11}+c_{13}\left\|\mathbb{A}^{-1} v\right\|^{p_{1}-1} \leq c_{11}+c_{13}\|v\|_{*}^{p_{1}-1}
$$

(we have used the Lipschitz continuity of $\mathbb{A}^{-1}$ with constant 1 and the chain (3.13) of continuous embeddings with dense images). So, we arrive at the estimate

$$
\left\|\mathbb{B}_{1}\left(\mathbb{A}^{-1} v\right)\right\|_{*} \leq c_{11}+c_{13}\|v\|_{*}^{p_{1}-1} \quad \text { for all } \quad v \in \mathbb{H}^{-2}(\Omega) .
$$

The following estimate is proved similarly:

$$
\left\|\mathbb{B}_{2}\left(\mathbb{A}^{-1} v\right)\right\|_{*} \leq c_{14}+c_{15}\|v\|_{*}^{p_{2}-1} \quad \text { for } \quad v \in \mathbb{H}^{-2}(\Omega) .
$$

Therefore, if $r$ is chosen so large that

$$
\left\|w_{1}\right\|_{*} \leq\left\|v_{0}\right\|_{*}+\mathrm{T}\left\|\mathbb{B}_{1}\left(\mathbb{A}^{-1} v_{0}\right)\right\|_{*} \leq\left\|v_{0}\right\|_{*}+\mathrm{T} c_{11}+\mathrm{T} c_{13}\left\|v_{0}\right\|_{*}^{p_{1}-1},
$$


we obtain

$$
\left\|w_{1}\right\|_{*} \leq \frac{r}{2}
$$

Now, we introduce the operator

$$
\mathbb{H}(v) \equiv w_{1}+\int_{0}^{t} d s G(v)(s),
$$

where $G(v)(s)$ is defined by (4.14). Then the integral equation (4.13) can be rewritten in the form

$$
v=\mathbb{H}(v) .
$$

We show that the operator $\mathbb{H}(v)$ defined by (4.21) acts from $\mathbb{V}_{r}$ to $\mathbb{V}_{r}$ and is a contraction on $\mathbb{V}_{r}$ for sufficiently large $r>0$ and sufficiently small $\mathrm{T}>0$.

First, we estimate the operator $G(v)(s)$ defined by (4.14) from above. Observe that $\mathbb{L}$ is bounded as an operator

$$
\mathbb{L} \equiv-\triangle: \mathbb{H}_{0}^{2}(\Omega) \subset \mathbb{H}_{0}^{1}(\Omega) \rightarrow \mathbb{H}^{-1}(\Omega) \subset \mathbb{H}^{-2}(\Omega) ;
$$

consequently, by (4.18) and (4.19), we obtain

$$
\begin{aligned}
\|G(v)\|_{*}(s) \leq\left\|v_{1}\right\|_{*}+c_{11} & +c_{13}\|v\|_{*}^{p_{1}-1}(s) \\
& +\int_{0}^{s} d \sigma\left[c_{16}\|v\|_{*}(\sigma)+c_{14}+c_{15}\|v\|_{*}^{p_{2}-1}(\sigma)\right] .
\end{aligned}
$$

Now we can estimate $\mathbb{H}(v)$ as follows:

$$
\begin{aligned}
\|\mathbb{H}(v)\| & \leq\left\|w_{1}\right\|_{*}+\mathrm{T}\left[\left\|v_{1}\right\|_{*}+c_{11}+c_{13}\|v\|^{p_{1}-1}\right]+\mathrm{T}^{2}\left[c_{16}\|v\|+c_{14}+c_{15}\|v\| \|^{p_{2}-1}\right] \\
& \leq \frac{r}{2}+\mathrm{T}\left[\left\|v_{1}\right\|_{*}+c_{11}+c_{13} r^{p_{1}-1}\right]+\mathrm{T}^{2}\left[c_{16} r+c_{14}+c_{15} r^{p_{2}-1}\right] \leq \frac{r}{2}+\frac{r}{2}=r
\end{aligned}
$$

if $\mathrm{T}>0$ is sufficiently small and $r>0$ is sufficiently large. Thus,

$$
\mathbb{H}: \mathbb{V}_{r} \rightarrow \mathbb{V}_{r} \text {. }
$$

We prove that $\mathbb{H}$ is a contraction on $\mathbb{V}_{r}$ for small $\mathrm{T}>0$ and large $r>0$. Indeed, let $v_{1}$, $v_{2} \in \mathbb{V}_{r}$. Using (iii) ${ }_{1}$ and (iv) $)_{2}$, we obtain

$$
\begin{aligned}
\left\|\mathbb{H}\left(v_{1}\right)-\mathbb{H}\left(v_{2}\right)\right\| \leq \mathrm{T}\left\|G\left(v_{1}\right)-G\left(v_{2}\right)\right\| \leq \mathrm{T}\left\|\mathbb{B}_{1}\left(\mathbb{A}^{-1} v_{1}\right)-\mathbb{B}_{1}\left(\mathbb{A}^{-1} v_{2}\right)\right\| \\
\quad+\mathrm{T}^{2}\left\|\mathbb{L} \mathbb{A}^{-1} v_{1}-\mathbb{L} \mathbb{A}^{-1} v_{2}\right\|+\mathrm{T}^{2}\left\|\mathbb{B}_{2}\left(\mathbb{A}^{-1} v_{1}\right)-\mathbb{B}_{2}\left(\mathbb{A}^{-1} v_{2}\right)\right\| \\
\leq \mathrm{T} c_{17} \operatorname{ess~sup}_{t \in[0, T]}^{\operatorname{ess}} \mu_{1}\left(\mathrm{R}_{1}\right)\left\|\mathbb{A}^{-1} v_{1}-\mathbb{A}^{-1} v_{2}\right\|_{1, p_{1}}+\mathrm{T}^{2} c_{17} \underset{t \in[0, \mathrm{~T}]}{\operatorname{ess} \sup \left\|\mathbb{A}^{-1} v_{1}-\mathbb{A}^{-1} v_{2}\right\|} \\
\quad+\mathrm{T}^{2} c_{17} \operatorname{esssup}_{t \in[0, \mathrm{~T}]} \mu_{2}\left(\mathrm{R}_{2}\right)\left\|\mathbb{A}^{-1} v_{1}-\mathbb{A}^{-1} v_{2}\right\|_{1, p_{2}},
\end{aligned}
$$

where

$$
\mathrm{R}_{1}=\max \left\{\left\|\mathbb{A}^{-1} v_{1}\right\|_{1, p_{1}},\left\|\mathbb{A}^{-1} v_{2}\right\|_{1, p_{1}}\right\}, \quad \mathrm{R}_{2}=\max \left\{\left\|\mathbb{A}^{-1} v_{1}\right\|_{1, p_{2}},\left\|\mathbb{A}^{-1} v_{2}\right\|_{1, p_{2}}\right\},
$$

and $\|\cdot\|_{1, p}$ stands for the norm in $\mathbb{W}_{0}^{1, p}(\Omega)$ for $p>2$. Using the continuous embedding

$$
\mathbb{H}_{0}^{2}(\Omega) \subset \mathbb{W}_{0}^{1, p}(\Omega) \text { for } N=3 \text { and } p=(2,6],
$$

we continue (4.24) to obtain

$$
\begin{aligned}
\left\|\mathbb{H}\left(v_{1}\right)-\mathbb{H}\left(v_{2}\right)\right\| & \leq c_{17} c_{18} \mathrm{~T} \mu_{1}\left(c_{18} \mathrm{R}\right)\left\|v_{1}-v_{2}\right\| \\
& +c_{17} \mathrm{~T}^{2}\left\|v_{1}-v_{2}\right\|+c_{19} c_{17} \mathrm{~T}^{2} \mu_{2}\left(c_{19} \mathrm{R}\right)\left\|v_{1}-v_{2}\right\| .
\end{aligned}
$$

We have used the Lipschitz continuity of $\mathbb{A}^{-1}$ with constant 1 . In inequality (4.25), $\mathrm{R}=\max \left\{\left\|v_{1}\right\|,\left\|v_{2}\right\|\right\}, c_{18}$ is the best constant for the embedding $\mathbb{H}_{0}^{2}(\Omega) \subset \mathbb{W}_{0}^{1, p_{1}}(\Omega)$ :

$$
\|w\|_{1, p_{1}} \leq c_{18}\|w\| \quad \text { for all } w \in \mathbb{H}_{0}^{2}(\Omega)
$$


and $c_{19}$ is the best constant for the embedding $\mathbb{H}_{0}^{2}(\Omega) \subset \mathbb{W}_{0}^{1, p_{2}}(\Omega)$ :

$$
\|w\|_{1, p_{2}} \leq c_{19}\|w\| \text { for all } w \in \mathbb{H}_{0}^{2}(\Omega) .
$$

Observe that $R \leq r$ because $v_{1}, v_{2} \in \mathbb{V}_{r}$. Now, we require that

$$
c_{18} c_{17} \mathrm{~T} \mu_{1}\left(c_{18} r\right)+c_{17} \mathrm{~T}^{2}+c_{17} c_{19} \mathrm{~T}^{2} \mu_{2}\left(c_{19} r\right) \leq \frac{1}{2} .
$$

This can be ensured by choosing $\mathrm{T}>0$ sufficiently small. As a result, we arrive at the inequality

$$
\left\|\mathbb{H}\left(v_{1}\right)-\mathbb{H}\left(v_{2}\right)\right\| \leq \frac{1}{2}\left\|v_{1}-v_{2}\right\| .
$$

By the contraction mapping theorem, the operator equation (4.22) has a unique solution $v(x, t) \in \mathbb{L}^{\infty}\left(0, \mathrm{~T} ; \mathbb{H}^{-2}(\Omega)\right)$.

Now, we prove that the solution $v(t) \in \mathbb{L}^{\infty}\left(0, \mathrm{~T} ; \mathbb{H}^{-1}(\Omega)\right)$ of the integral equation (4.13) can be extended to a maximal interval $\left[0, \mathrm{~T}_{0}\right)$, where either $\mathrm{T}_{0}=+\infty$ or $\mathrm{T}_{0}<+\infty$; in the latter case,

$$
\underset{t \uparrow T_{0}}{\limsup }\|v\|_{*}(t)=+\infty
$$

Indeed, consider the norm

$$
\psi(\mathrm{T}) \equiv \operatorname{ess~sup}_{t \in[0, \mathrm{~T}]}\|v(t)\|_{*}(t) .
$$

As a function of $\mathrm{T}$, the function $\psi(\mathrm{T})$ is monotone nondecreasing. Therefore, as $\mathrm{T} \uparrow \mathrm{T}_{0}$, $\psi(\mathrm{T})$ has a limit, which is either finite or infinite. Suppose it is finite, and let $\mathrm{T}^{\prime} \in\left(0, \mathrm{~T}_{0}\right)$. Consider the following integral equations:

$$
v\left(\mathrm{~T}^{\prime}\right)=v_{0}+\int_{0}^{\mathrm{T}^{\prime}} d s \bar{G}(v)(s), \quad \bar{G}(v)(s)=G(v)(s)+\mathbb{B}_{1}\left(\mathbb{A}^{-1} v_{0}\right),
$$

where $G(v)(s)$ is defined by (4.14), and

$$
v\left(\mathrm{~T}^{\prime}+t\right)=v\left(\mathrm{~T}^{\prime}\right)+\int_{\mathrm{T}^{\prime}}^{\mathrm{T}^{\prime}+t} d s \bar{G}(v)(s), \quad t>0 .
$$

We introduce the function $w(t)=v\left(\mathrm{~T}^{\prime}+t\right)$ and make the change of variables $\sigma=s-\mathrm{T}^{\prime}$ in the last integral. This results in the equation

$$
w(t)=v\left(\mathrm{~T}^{\prime}\right)+\int_{0}^{t} d \sigma \bar{G}(w)(\sigma), \quad t>0 .
$$

We observe that

$$
\|v\|_{*}\left(\mathrm{~T}^{\prime}\right) \leq C<+\infty, \quad \mathrm{T}^{\prime} \in\left(0, \mathrm{~T}_{0}\right) .
$$

Since equation (4.29) is of the form (4.13), there exists a time moment $\mathrm{T}^{*}=\mathrm{T}^{*}\left(\mathrm{~T}^{\prime}\right)$ such that (4.29) has a solution on the interval $t \in\left(0, \mathrm{~T}^{*}\right)$. By (4.30), the function $\mathrm{T}^{*}=\mathrm{T}^{*}\left(\mathrm{~T}^{\prime}\right)$ has a positive minimum, which we redenote by $T^{*}$. Now, we take $T^{\prime}=T_{0}-T^{*} / 2$ for the role of new $\mathrm{T}^{\prime}$. So, problem (4.29) admits a solution on the interval $t \in\left(0, \mathrm{~T}^{*}\right)$. We substitute in (4.29) the expression for $v\left(\mathrm{~T}^{\prime}\right)$ given by (4.27), obtaining

$$
v\left(t_{0}\right)=v_{0}+\int_{0}^{t_{0}} d s \bar{G}(v)(s), \quad t_{0}=\mathrm{T}^{\prime}+t, t \in\left(0, \mathrm{~T}^{*}\right) .
$$

By the above, there is a unique solution of problem (4.31) on the interval $t_{0} \in\left(0, \mathrm{~T}^{\prime}+\mathrm{T}^{*}\right)$. By the choice $\mathrm{T}^{\prime}=\mathrm{T}_{0}-\mathrm{T}^{*} / 2$, we have $t_{0} \in\left(0, \mathrm{~T}_{0}+\mathrm{T}^{*} / 2\right)$. So, employing the same algorithm of extension in time, we draw the conclusion that $\mathrm{T}_{0}=+\infty$, contradicting our assumption that $\mathrm{T}_{0}<+\infty$. It follows that

$$
\lim _{\mathrm{T} \uparrow \mathrm{T}_{0}} \psi(\mathrm{T})=+\infty
$$


This immediately implies the relation

$$
\limsup _{t \uparrow T_{0}}\|v\|_{*}(t)=+\infty .
$$

We prove that the solution $v(x, t) \in \mathbb{L}^{\infty}\left(0, \mathrm{~T} ; \mathbb{H}^{-2}(\Omega)\right)$ belongs to $\mathbb{C}^{(2)}\left(0, \mathrm{~T} ; \mathbb{H}^{-2}(\Omega)\right)$. We use the so-called "bootstrap" method. First, we show that

$$
v(x, t) \in \mathbb{C}\left(0, \mathrm{~T} ; \mathbb{H}^{-2}(\Omega)\right) .
$$

Indeed, we have

$$
\left\|v\left(t_{1}\right)-v\left(t_{2}\right)\right\|_{*} \leq \int_{t_{1}}^{t_{2}} d s\|\bar{G}(v)\|_{*}(s) \leq\|\bar{G}(v)\||| t_{1}-t_{2} \mid \rightarrow 0 \quad \text { as } \quad t_{1} \rightarrow t_{2}
$$

for all $t_{1}, t_{2} \in[0, \mathrm{~T}]$. Consequently, $v(x, t) \in \mathbb{C}\left(0, \mathrm{~T} ; \mathbb{H}^{-2}(\Omega)\right)$. Now, we show that $v(x, t) \in \mathbb{C}^{(1)}\left(0, \mathrm{~T} ; \mathbb{H}^{-2}(\Omega)\right)$. Indeed, for this we must prove that

$$
G(v)(s) \in \mathbb{C}\left(0, \mathrm{~T} ; \mathbb{H}^{-2}(\Omega)\right) .
$$

Since $v(x, t) \in \mathbb{C}\left(0, \mathrm{~T} ; \mathbb{H}^{-2}(\Omega)\right)$ and $\mathbb{A}^{-1}$ is Lipschitz continuous with constant 1 , we have

$$
\left\|\mathbb{A}^{-1} v\left(t_{1}\right)-\mathbb{A}^{-1} v\left(t_{2}\right)\right\| \leq\left\|v\left(t_{1}\right)-v\left(t_{2}\right)\right\|_{*} \rightarrow 0 \quad \text { as } \quad t_{1} \rightarrow t_{2}
$$

for all $t_{1}, t_{2} \in[0, \mathrm{~T}]$. Thus, $\mathbb{A}^{-1} v \in \mathbb{C}\left(0, \mathrm{~T} ; \mathbb{H}_{0}^{2}(\Omega)\right)$. On the other hand, by (iii) $)_{1}$ and (iv) $)_{2}$ for $\mathbb{B}_{1}(\cdot)$ and $\mathbb{B}_{2}(\cdot)$, we have

$$
\left\|\mathbb{B}_{1}\left(\mathbb{A}^{-1} v\left(t_{1}\right)\right)-\mathbb{B}_{1}\left(\mathbb{A}^{-1} v\left(t_{2}\right)\right)\right\|_{*} \leq c_{21} \mu_{1}\left(c_{20} \mathrm{R}\right)\left\|\mathbb{A}^{-1} v\left(t_{1}\right)-\mathbb{A}^{-1} v\left(t_{2}\right)\right\| \rightarrow 0
$$

as $t_{1} \rightarrow t_{2}$ for all $t_{1}, t_{2} \in[0, \mathrm{~T}]$. Thus,

$$
\mathbb{B}_{1}\left(\mathbb{A}^{-1} v(t)\right) \in \mathbb{C}\left(0, \mathrm{~T} ; \mathbb{H}^{-2}(\Omega)\right) .
$$

Similarly,

$$
\mathbb{B}_{2}\left(\mathbb{A}^{-1} v(t)\right) \in \mathbb{C}\left(0, \mathrm{~T} ; \mathbb{H}^{-2}(\Omega)\right)
$$

Finally,

$$
\mathbb{L} \mathbb{A}^{-1} v(t) \in \mathbb{C}\left(0, \mathrm{~T} ; \mathbb{H}^{-2}(\Omega)\right)
$$

because $\mathbb{L} \equiv-\triangle$ is linear and continuous as an operator acting as indicated below:

$$
\mathbb{L} \equiv-\triangle: \mathbb{H}_{0}^{2}(\Omega) \subset \mathbb{H}_{0}^{1}(\Omega) \rightarrow \mathbb{H}^{-1}(\Omega) \subset \mathbb{H}^{-2}(\Omega)
$$

Therefore,

Consequently,

$$
G(v)(s) \in \mathbb{C}\left(0, \mathrm{~T} ; \mathbb{H}^{-2}(\Omega)\right) .
$$

$$
\mathbb{H}(v) \in \mathbb{C}^{(1)}\left(0, \mathrm{~T} ; \mathbb{H}^{-2}(\Omega)\right),
$$

and by (4.22) we obtain

$$
v(x, t) \in \mathbb{C}^{(1)}\left(0, \mathrm{~T} ; \mathbb{H}^{-2}(\Omega)\right) .
$$

Now, we show that, in combination with the definition (4.14) of $G(v)(s)$, this implies

$$
\mathbb{H}(v) \in \mathbb{C}^{(2)}\left(0, \mathrm{~T} ; \mathbb{H}^{-2}(\Omega)\right) .
$$

To this end, we need to know that

$$
\mathbb{B}_{1}\left(\mathbb{A}^{-1} v(t)\right) \in \mathbb{C}^{(1)}\left(0, \mathrm{~T} ; \mathbb{H}^{-2}(\Omega)\right) .
$$

By the chain rule for the Fréchet derivative (Theorem 1), we have

$$
\mathbb{A}^{-1} v(t) \in \mathbb{C}^{(1)}\left(0, \mathrm{~T} ; \mathbb{H}_{0}^{2}(\Omega)\right),
$$

because $\mathbb{A}^{-1}: \mathbb{H}^{-2}(\Omega) \rightarrow \mathbb{H}_{0}^{2}(\Omega)$ is a bounded linear operator. By (iii) 1 , the operator

$$
\mathbb{B}_{1}(w) \equiv-\operatorname{div}\left(\phi_{1}(x,|\nabla w|) \nabla w\right): \mathbb{W}_{0}^{1, p_{1}}(\Omega) \rightarrow \mathbb{W}^{-1, p_{1}^{\prime}}(\Omega)
$$


is Fréchet differentiable. Applying Theorem 1, we obtain

$$
\mathbb{B}_{1}\left(\mathbb{A}^{-1} v(t)\right) \in \mathbb{C}^{(1)}\left(0, \mathrm{~T} ; \mathbb{H}^{-2}(\Omega)\right) .
$$

Thus,

but then

$$
G(v)(s) \in \mathbb{C}^{(1)}\left(0, \mathrm{~T} ; \mathbb{H}^{-2}(\Omega)\right),
$$

$$
\mathbb{H}(v) \in \mathbb{C}^{(2)}\left(0, \mathrm{~T} ; \mathbb{H}^{-2}(\Omega)\right) .
$$

Consequently, the operator identity (4.22) shows that

$$
v(x, t) \in \mathbb{C}^{(2)}\left(0, \mathrm{~T} ; \mathbb{H}^{-2}(\Omega)\right) .
$$

But

$$
u=\mathbb{A}^{-1} v,
$$

and the chain rule for Fréchet derivatives (Theorem 1) implies

$$
u(x, t) \in \mathbb{C}^{(2)}\left([0, \mathrm{~T}] ; \mathbb{H}_{0}^{2}(\Omega)\right) .
$$

Thus we have proved the following theorem.

Theorem 2. Suppose that conditions $(\mathrm{i})_{1}-(\mathrm{iii})_{1}$ and (i $)_{2}$, (ii) $)_{2},(\text { iv })_{2}$ are fulfilled; then for every $u_{0}(x), u_{1}(x) \in \mathbb{H}_{0}^{2}(\Omega)$ there exists $\mathrm{T}_{0}=\mathrm{T}_{0}\left(u_{0}, u_{1}\right)>0$ such that problem (2.11)(2.13) admits a strong generalized solution $u(x, t) \in \mathbb{C}^{(2)}\left(\left[0, \mathrm{~T}_{0}\right) ; \mathbb{H}_{0}^{2}(\Omega)\right)$ understood in the sense of Definition 3. Moreover, either $\mathrm{T}_{0}=+\infty$ or $\mathrm{T}_{0}<+\infty$, and in the latter case we have

$$
\limsup _{t \uparrow T_{0}}\|\mathbb{A} u\|_{*}(t)=+\infty, \quad \mathbb{A} \equiv \triangle^{2}-\triangle .
$$

Remark 6. We discuss formula (4.32). Observe that

$$
\begin{aligned}
\|\mathbb{A} u\|_{*}(t) & =\sup _{w \in \mathbb{H}_{0}^{2}(\Omega),\|w\| \leq 1}|\langle\mathbb{A} u, w\rangle| \\
& \leq \sup _{w \in \mathbb{H}_{0}^{2}(\Omega),\|w\| \leq 1}\left|\int_{\Omega}(\nabla u, \nabla w) d x+\int_{\Omega} \Delta u \triangle w d x\right| \\
& \leq 2 \sup _{w \in \mathbb{H}_{0}^{2}(\Omega),\|w\| \leq 1} \Phi^{1 / 2}[u](t) \Phi^{1 / 2}[w] \leq c_{22} \Phi^{1 / 2}[u](t),
\end{aligned}
$$

where $\Phi^{1 / 2}[u](t)$ is defined by (3.20). Next, we have the following lower estimate:

$$
\|\mathbb{A} u\|_{*}(t)\|u\|(t) \geq\langle\mathbb{A} u(t), u(t)\rangle=2 \Phi(t) .
$$

On the other hand,

$$
\|u\|^{2}=\int_{\Omega}|\triangle u|^{2} d x
$$

and the definition (3.20) implies the upper estimate $\|u\|^{2}(t) \leq 2 \Phi(t)$. Combined with (3.34), this yields the inequality

$$
\sqrt{2} \Phi^{1 / 2}[u](t)\|\mathbb{A} u\|_{*}(t) \geq 2 \Phi(t) \Rightarrow\|\mathbb{A} u\|_{*}(t) \geq \sqrt{2} \Phi^{1 / 2}[u](t) .
$$

We have arrived at the two-sided estimate

$$
\sqrt{2} \Phi^{1 / 2}[u](t) \leq\|\mathbb{A} u\|_{*}(t) \leq c_{22} \Phi^{1 / 2}[u](t) .
$$

So, expression (4.32) is equivalent to

$$
\limsup _{t \uparrow T_{0}} \Phi[u](t)=+\infty,
$$

where the functional $\Phi[u](t)$ is defined by (3.20) . 
Thus, Theorem 2 and the results of $\S 3$ imply the following statement, which is the principal result of the paper.

Theorem 3. Suppose that functions $\phi_{1}(x, s)$ and $\phi_{2}(x, s)$ satisfy $(\mathrm{i})_{1}-(\mathrm{iii})_{1}$ and $(\mathrm{i})_{2}-$ (iv) $)_{2}, u_{0}, u_{1} \in \mathbb{H}_{0}^{2}(\Omega)$, and the following conditions are fulfilled:

$$
\begin{aligned}
\vartheta & >2+2 q_{1}, \quad q_{1}=p_{1}-2, \\
\int_{\Omega} \mathcal{F}\left(x,\left|\nabla u_{0}\right|\right) d x & \geq \frac{1}{2} \int_{\Omega}\left|\nabla u_{1}\right|^{2} d x+\frac{1}{2} \int_{\Omega}\left|\triangle u_{1}\right|^{2} d x+\frac{1}{2} \int_{\Omega}\left|\nabla u_{0}\right|^{2} d x, \\
\mathcal{F}(x, s) & =\int_{0}^{s} d \sigma \sigma \phi_{2}(x, \sigma), \\
\Phi^{\prime}(0) & >\left(\frac{\beta}{\alpha-1} \Phi_{0}^{2}+\frac{2 \gamma}{2 \alpha-q_{1}-2} \Phi_{0}^{2+q_{1}}\right)^{1 / 2} .
\end{aligned}
$$

Then the existence time for the strong generalized solution is positive and bounded above:

$$
\mathrm{T}_{0} \in\left(0, \mathrm{~T}_{1}\right], \quad \mathrm{T}_{1}=\frac{\Phi_{0}^{1-\alpha}}{A}>0,
$$

and

$$
\limsup _{t \uparrow T_{0}} \Phi(t)=+\infty
$$

where

$$
\begin{aligned}
\Phi(t) & \equiv \frac{1}{2} \int_{\Omega}|\nabla u|^{2} d x+\frac{1}{2} \int_{\Omega}|\triangle u|^{2} d x \\
\Phi_{0} & =\Phi(0)=\frac{1}{2} \int_{\Omega}\left|\nabla u_{0}\right|^{2} d x+\frac{1}{2} \int_{\Omega}\left|\triangle u_{0}\right|^{2} d x \\
\Phi^{\prime}(0) & =\int_{\Omega}\left(\nabla u_{1}, \nabla u_{0}\right) d x+\int_{\Omega} \triangle u_{1} \triangle u_{0} d x \\
\alpha & =\frac{1}{2}\left(1+\frac{\vartheta}{2}-\varepsilon_{0}\right), \quad \beta=\frac{c_{7}}{\varepsilon_{0}}, \quad \gamma=\frac{c_{6}}{\varepsilon_{0}}, \quad \varepsilon_{0} \in\left(0, \frac{\vartheta-2-2 q_{1}}{2}\right), \\
A^{2} & \equiv(\alpha-1)^{2} \Phi_{0}^{-2 \alpha}\left(\Phi^{\prime}(0)\right)^{2}-\beta(\alpha-1) \Phi_{0}^{-2 \alpha} \Phi_{0}^{2}-\frac{2 \gamma(\alpha-1)}{\delta} \Phi_{0}^{-\delta \alpha} \Phi_{0}^{\delta}>0, \\
\delta & =1+\frac{1+q_{1}-\alpha}{1-\alpha}
\end{aligned}
$$

and $\varepsilon_{0}$ provides the minimum for the function

$$
Q_{0}=Q_{0}(\varepsilon) \equiv \frac{\beta}{\alpha-1}+\frac{2 \gamma}{2 \alpha-q_{1}-2} \Phi_{0}^{q_{1}}
$$

under the condition $\varepsilon_{0} \in\left(0,\left(\vartheta-2-2 q_{1}\right) / 2\right)$.

\section{$\S 5$. CONCLUSION}

It should be noted that, in the present paper, we have considered localized "sources" (described by $\phi_{2}(x, s)$ ) and "drains" (described by $\phi_{1}(x, s)$ ) in plasma This is important from a physical viewpoint because sources and drains in real plasma are localized indeed. Much as in this paper, it is possible to derive the equation for nonlinear ion-sound waves in "magnetized" plasma. However, that equation is of the fourth order in time and involves a nonlinear elliptic operator at the second derivative with respect to time. As far as I know, such equations have not been investigated as yet. 


\section{REFERENCES}

[1] H. Gajewski, K. Gröger, and K. Zacharias, Nichtlineare Operatorgleichungen und Operatordifferentialgleichungen, Math. Lehrbucher und Monogr., II Abteilung, Math. Monogr., Bd. 38, Akademie-Verlag, Berlin, 1974. MR0636412 (58:30524a)

[2] V. A. Galaktionov and S. I. Pokhozhaev, Third-order nonlinear dispersion equations: shock waves, rarefaction waves, and blow-up waves, Zh. Vychisl. Mat. Mat. Fiz. 48 (2008), no. 10, 18191846; English transl., Comput. Math. Math Phys. 48 (2008), no. 10, 1784-1810. MR2493769 (2009k:35031)

[3] V. K. Kalantarov and O. A. Ladyzhenskaya, Formation of collapses in quasilinear equations of parabolic and hyperbolic types, Zap. Nauchn. Sem. Leningrad. Otdel. Mat. Inst. Steklov. (LOMI) 69 (1977), 77-102; English transl. in J. Soviet Math. 10 (1978), no. 1. MR0604036 (58:29269)

[4] M. A. Krasnosel'skiı̌, Topological methods in the theory of nonlinear integral equations, Costekhizdat, Moscow, 1956; English transl., The Macmillan Co., New York, 1964. MR0096983 (20:3464) MR0159197 (28:2414)

[5] N. V. Krylov, Lectures on elliptic and parabolic equations in Hölder spaces, Nauchn. Kniga, Novosibirsk, 1998; Translated from the 1996 English original, Grad. Stud. in Math., vol. 12, Amer. Math. Soc., Providence, RI, 1996. MR 1657890 (99g:35002) MR1406091 (97i:35001)

[6] L. D. Landau and E. M. Lifshitz, Theoretical physics. Vol. 8. Electrodynamics of continuous media, 3rd ed., Nauka, Moscow, 1992; English transl., Pergamon Press, Oxford, 1960. MR1330694 (95m:00001) MR0121049 (22:11796)

[7] , Theoretical physics. Vol. 10. Physical kinetics, Fizmatlit, Moscow, 2001. (Russian)

[8] È. Mitidieri and S. I. Pokhozhaev, A priori estimates and the absence of solutions of nonlinear partial differential equations and inequalities, Tr. Mat. Inst. Steklova 234 (2001), 1-384; English transl., Proc. Steklov Inst. Math. 2001, no. 3 (234), 1-362. MR.1879326 (2005d:35004)

[9] A. A. Samarskiı̌, V. A. Galaktionov, S. P. Kurdyumov, and A. P. Mikhal̆lov, Peaking modes in problems for quasilinear parabolic equations, Nauka, Moscow, 1987; English transl., Blow-up in quasilinear parabolic equations, Gruyter Exp. in Math., vol. 19, Walter de Gruyter, Berlin, 1995. MR0919951 (89a:35002) MR.1330922 (96b:35003)

[10] A. G. Sveshnikov, A. B. Al'shin, M. O. Korpusov, and Yu. D. Pletner, Linear and nonlinear equations of the Sobolev type, Fizmatlit, 2007, 734 pp. (Russian)

[11] I. E. Tamm, Foundations of the electricity theory, Nauka, Moscow, 1989. (Russian)

[12] L. Gasinski and N. S. Papageorgiou, Nonlinear analysis, Ser. in Math. Anal. Appl., vol. 9, Chapman and Hall, Boca Raton, FL, 2006. MR2168068 (2006e:47001)

[13] H. A. Levine, Some nonexistence and instability theorems for solutions of formally parabolic equations of the form $P u_{t}=-A u+\mathcal{F}(u)$, Arch. Rational Mech. Anal. 51 (1973), 371-386. MR0348216 (50:714)

[14] Instability and nonexistence of global solutions to nonlinear wave equations of the form $P u_{t t}=-A u+\mathcal{F}(u)$, Trans. Amer. Math. Soc. 192 (1974), 1-21. MR0344697 (49:9436)

Department of Physics, Lomonosov Moscow State University, Moscow, Russia

E-mail address: korpusov@gmail.com

Received 27/MAY/2010

Translated by S. V. KISLYAKOV 